\title{
Un enfoque dinámico para el análisis de la pobreza e indigencia en Argentina
}

\author{
Valentina Bonifacio \\ Tesis de Maestría \\ Maestría en Economía \\ Universidad Nacional de la Plata
}

Directora: Roxana Maurizio 


\title{
Un enfoque dinámico para el análisis de la pobreza e indigencia en Argentina
}

\begin{abstract}
Resumen
En Argentina, luego de la crisis macroeconómica del 2001, comienza una década de reducción de la pobreza. Sin embargo, desde un análisis dinámico, se observa que a pesar de que se redujeron los niveles agregados, hay un porcentaje significativo de hogares que entran y salen de la pobreza constantemente. El objetivo de este trabajo es analizar las tasas de salida de la pobreza y la indigencia para el período 2003-2014, e identificar a qué tipo de eventos se asocian estas transiciones. Los eventos pueden estar relacionados con el mercado laboral, con cambios demográficos o con transferencias desde el sector público. Los resultados sugieren que las tasas de salida están asociadas principalmente a factores laborales, y que existe una brecha en las tasas de salida de la pobreza de los hogares con y sin menores. Asimismo, se aprecia que hubo un incremento en las transferencias gubernamentales, en especial para los hogares con presencia de menores, pero aun así dos tercios de ellos no lograron salir de la pobreza. No obstante, la obtención de alguna transferencia se asocia con una mayor probabilidad de salida de la indigencia.
\end{abstract}

Clasificación JEL I32

Palabras claves: tasas de salida, pobreza, indigencia, transferencias.

\begin{abstract}
After the 2001 macroeconomic crisis, Argentina was followed by a poverty reduction decade. However, from a dynamics analysis, it is shown that although aggregate levels were reduced, a significant percentage of households are constantly entering and existing poverty. The objective of this paper is to analyze poverty and indigence exit rates for the period 2003-2014, identifying the type of events associated with these transitions. These events may be related to the labor market, demographic changes or public monetary transferences. The results suggest that exit rates are mainly associated with labor factors, and that there is a gap between the poverty exit rates of households with and without children. Likewise, it is shown that there was also an increase in government transfers, especially for households with children, but despite of this, two-thirds of them were unable to escape poverty. However, receiving public transference is associated with a greater probability of leaving indigence.
\end{abstract}

JEL Clasification: I32

Key words: exit rates, poverty, indigence, public transferences 


\section{$\underline{\text { Tabla de contenido }}$}

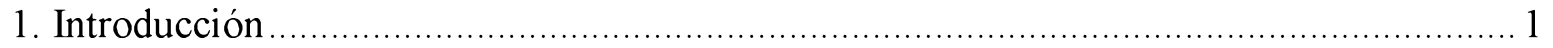

2. Marco teórico

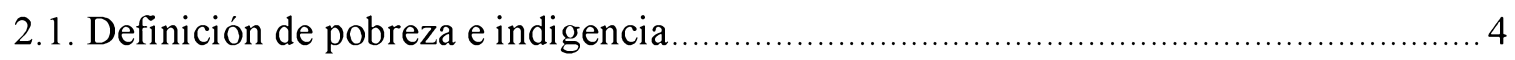

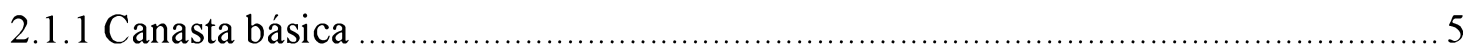

2.2. Evidencia empírica sobre la dinámica de la pobreza ............................................. 6

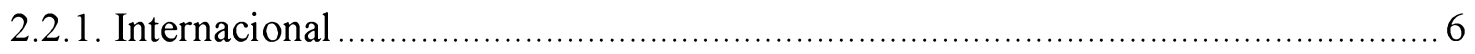

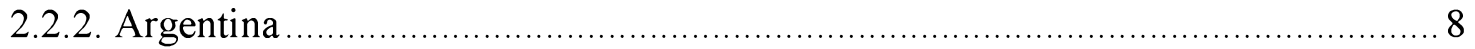

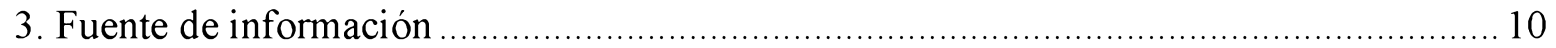

3.1. Encuesta Permanente de Hogares ....................................................................... 10

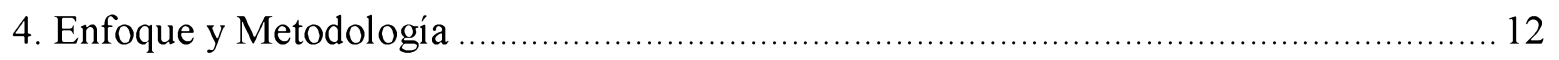

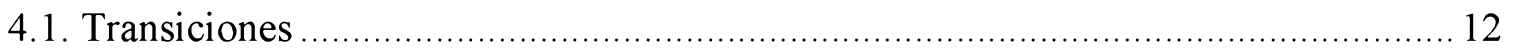

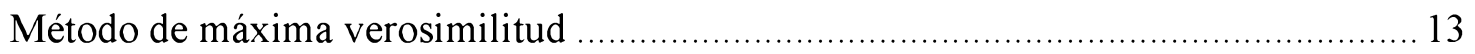

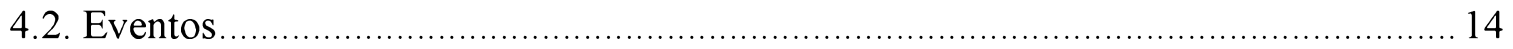

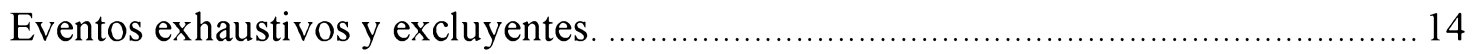

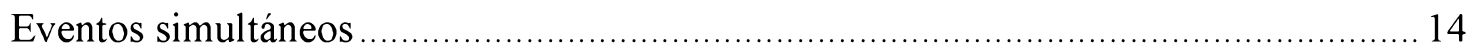

4.3 Descomposición de las probabilidades de transición.............................................. 14

5. Evolución de pobreza e indigencia y perfiles de los jefes de hogares............................ 16

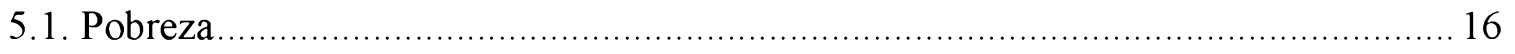

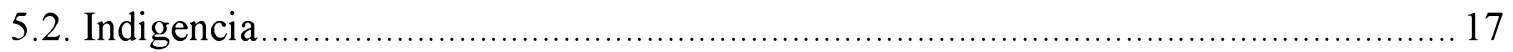

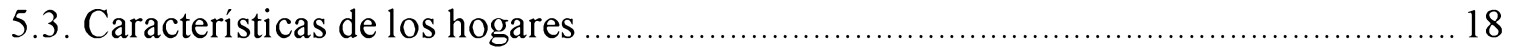

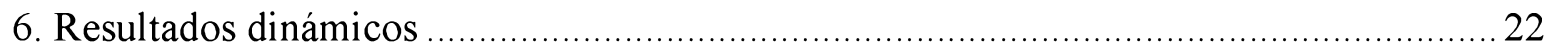

6.1. Tasas de entrada y salida de la pobreza e indigencia ............................................. 22

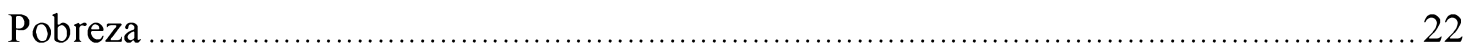

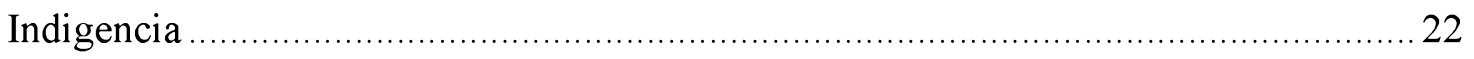

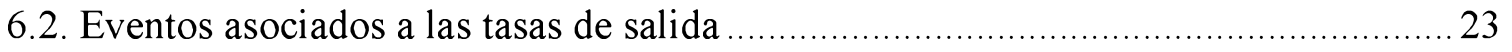

Pobreza ………

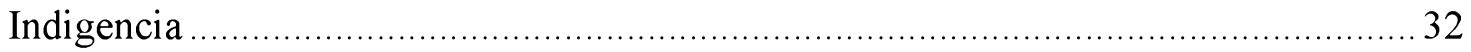




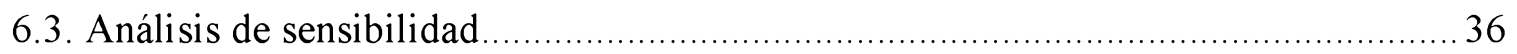

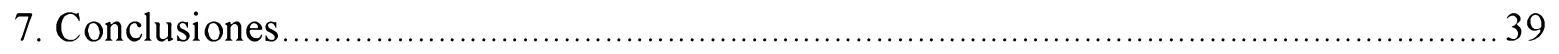

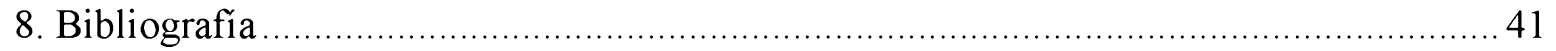

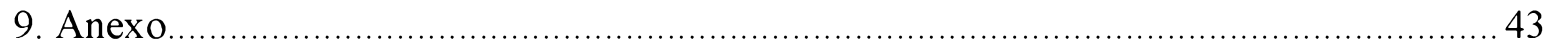

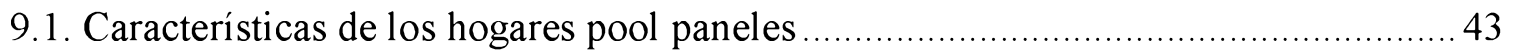

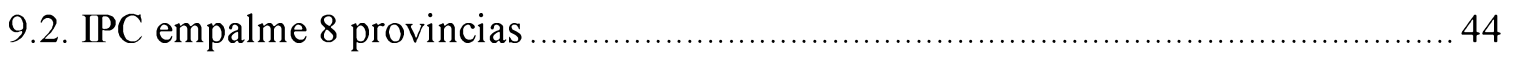




\section{Introducción}

Existe consenso de que la pobreza es un mal social, tanto por el impacto que genera las personas que la sufren como también por las potenciales consecuencias sobre variables económicas y sociales. Desde la problemática individual, la pobreza se traduce en carencias de requerimientos mínimos para alcanzar una calidad de vida aceptable. Asimismo, deficiencias en la alimentación y en la salud limitan la productividad y, por lo tanto, la capacidad de obtener el ingreso necesario para vivir. La pobreza también incide en la educación de la familia, ya que muchos de ellos se ven obligados a trabajar para aportar ingresos al hogar, abandonando la educación, y perjudicando su desarrollo futuro. Este círculo vicioso aumenta la probabilidad de que las familias estén destinadas a esta condición. Por otro lado, desde la problemática social, las privaciones individuales de cada hogar pueden generar problemas de salud pública, inseguridad e incluso inestabilidad democrática. Finalmente, desde una perspectiva económica, la pobreza puede condicionar seriamente la posibilidad de acumular capital humano y otros factores productivos, y afectar negativamente las perspectivas de crecimiento económico (Gasparini et al (2013)). Por lo tanto, los motivos para reducir la pobreza son múltiples.

De esta manera, es relevante analizar la naturaleza y la intensidad de la dinámica de la pobreza, tanto para evaluar los logros y debilidades de las políticas de protección social, como para orientar futuras líneas de acción de la política pública. El análisis estático es útil para el monitoreo general, pero brinda información limitada, ya que sólo revela los niveles agregados de pobreza e indigencia. Aún cuando la trayectoria muestra una tendencia decreciente, existen hogares que entran y salen de la pobreza constantemente (Cantó et al 2006, 2009).

En este sentido, existe un enfoque dinámico que se basa en analizar las tasas de entrada y salida de la pobreza a partir de observar a un mismo hogar en diferentes momentos del tiempo. Algunos estudios como el de Jenkins y Schluter (2003), han complementado este enfoque asociando episodios a dichas transiciones, que permiten comprender si éstas están relacionadas con eventos del mercado laboral, cambios en la composición del hogar o con políticas públicas específicas. 
Las privaciones materiales que pueda tener un hogar dependen, entre muchos otros factores, de la composición dentro del hogar, dado que todos los integrantes tienen necesidades que satisfacer, pero no todos tienen la posibilidad de generar ingresos. De aquí, la distinción más relevante es entre los hogares con y sin presencia de menores. En principio, las familias con menores tienen más necesidades que completar y menos potenciales trabajadores que generen ingresos, bajo el supuesto de que los menores de 18 años no trabajan. Por lo tanto, este grupo de hogares son más vulnerables a caer en un estado de pobreza. (Beccaria y Maurizio (2006), Beccaria et al (2011, 2013, 2015)).

En Argentina, los mayores niveles de pobreza en base a la información histórica disponible, se registran a comienzo del nuevo siglo como consecuencia de la crisis macroeconómica, lo cual generó que alrededor del $60 \%$ de las personas se encuentren en una situación de la pobreza. Luego de este nivel máximo, el país experimenta un crecimiento económico que reduce los niveles agregados al 35\% en el año 2014 .

Sin embargo, hay que tener en cuenta que este nivel de pobreza fue extraordinario, consecuencia del abandono del modelo de convertibilidad ${ }^{1}$. La deuda acumulada en este régimen se tornó insostenible, finalizando el período con una devaluación de la moneda local de más del 300\% en un lapso corto de tiempo. El reacomodamiento de las variables monetarias, ocasionó un shock económico, que se vio reflejado en aumentos de precios y en la reducción de los ingresos nominales, dejando a gran parte de los hogares por debajo de la línea de la pobreza. A pesar de los daños que causó la devaluación, posteriormente, la nueva configuración de los precios relativos apoyada en la política de mantenimiento de tipo de cambio real, fue un factor preponderante en la reversión de la fase recesiva, generando aumento de la demanda laboral con una elasticidad empleo-producto que se mantuvo elevada en los próximos años (Beccaria et. al. 2005). En este contexto, se enmarca una década de reducción de pobreza.

El objetivo de esta investigación es estudiar las transiciones de los hogares en torno a la situación de pobreza, específicamente analizar las tasas de salida de la pobreza y de la

\footnotetext{
${ }^{1}$ A partir de la ley 23.928 sancionada en 1991, se declara una relación cambiaria fija entre la moneda nacional y el peso estadounidense a razón de un dólar a un peso (plan de convertibilidad). La emisión monetaria estaba restringida al respaldo de las reservas del Tesoro Nacional, por lo cual se debía mantener disciplina fiscal sólida.
} 
indigencia, asociadas a distintos eventos, según la composición familiar del hogar, para Argentina durante el período 2003-2014, distinguiendo en tres sub períodos de diferente reducción de pobreza: 2003 a 2006, 2008 a 2011 y 2011 a 2014.

Para determinar el nivel de pobreza se utiliza el enfoque de ingresos, por el cual se define que un hogar es pobre cuando los ingresos son insuficientes para cubrir las necesidades básicas de sus integrantes. Por lo tanto, hay dos factores que intervienen en esta definición: la fuente de ingresos (laboral o no laboral), y la composición del hogar. Con el fin caracterizar dichas transiciones, se clasifican en eventos laborales y no laborales a los cambios relacionados al primer factor, y eventos demográficos a los cambios ocasionados en el segundo factor.

La pregunta de investigación es la siguiente:

¿Cuál es la probabilidad de salida de la pobreza y de la indigencia asociada a determinado evento para cada tipo de hogar? En especial, se profundiza sobre los eventos que involucren transferencias gubernamentales. Asimismo, se realiza la descomposición de dicha probabilidad total en:

- La probabilidad de ocurrencia del "evento" en el hogar

- La probabilidad de que el hogar transite dentro y fuera del estado de pobreza condicional a la ocurrencia del evento.

Existe una amplia literatura sobre la incidencia y características de la pobreza en Argentina, pero la mayoría abordan el tema desde un análisis estático, y pocos se remiten al análisis dinámico. Distinguir entre las tasas de entrada y salida, permite detectar si la pobreza en un determinado momento se asocia a los altos flujos de entrada o a la duración más larga de los que ya están dentro de ella. Además, identificando cuales son los episodios que se asocian a dichas transiciones, es posible mejorar el diseño de políticas públicas con el fin de reducir las tasas de entrada y elevar las tasas de salida.

El trabajo se estructura de la siguiente manera. En la próxima sección se presenta el marco teórico de la investigación, lo cual incluye las definiciones de pobreza e indigencia y la evidencia empírica sobre estudios de la dinámica de la pobreza tanto a nivel internacional 
como nacional. En la tercera sección se expone la fuente de información utilizada, y en la cuarta la metodología del trabajo. Luego, en la quinta sección se realiza un análisis descriptivo sobre la evolución de la tasa de pobreza e indigencia y sobre los perfiles de los jefes de hogares. En la sexta, se exponen los resultados de las tasas de salida para los hogares pobres e indigentes. En la séptima sección se presentan las conclusiones del trabajo junto con las recomendaciones, y finalmente en la octava y novena sección, se presentan la bibliografía y anexo, respectivamente.

\section{Marco teórico}

\subsection{Definición de pobreza e indigencia}

La definición de pobreza (extrema y moderada) adoptada es la oficializada por el Instituto Nacional de Estadística y Censos (INDEC). Esta definición se rige según el enfoque de los ingresos. Un hogar es considerado pobre si sus ingresos monetarios son inferiores al valor de la canasta de bienes y servicios, necesaria para cubrir los requerimientos básicos alimentarios (determinadas por los requerimientos calóricos requeridos para vivir en combinación con los patrones de consumo de cada población) y otros bienes y servicios. Esta concepción de la pobreza es absoluta y está asociada a un conjunto de carencias específicas la cual es invariante al nivel de desarrollo de la sociedad en la que se miden las privaciones. En contraste, en países, donde el nivel de desarrollo es mayor, particularmente en los países europeos, cobra sentido tomar el criterio de pobreza relativa, por ejemplo, clasificar como pobres a los ciudadanos que tengan un ingreso $50 \%$ inferior a la media.

Para la pobreza extrema o indigencia, se toma sólo la canasta básica de alimentos. Es importante aclarar que la indigencia es un subconjunto de la pobreza. En otras palabras, son aquellos hogares que no llegan a cubrir al menos los requerimientos básicos alimenticios.

La elección de tomar una definición basada meramente en variables monetarias tiene ventajas y desventajas. Entre las ventajas se destaca la sencillez de cálculo, ya que se requieren menos variables que al estudiar otras definiciones de pobreza, tal como la multidimensional. Pero aun así hay que advertir el problema de identificación de los casos que se encuentran alrededor de la línea de la pobreza. Dada que la pobreza es binaria, los hogares pueden cruzar esta línea con cambios pequeños en el ingreso sin cambiar 
sustancialmente sus costumbres o su estatus social. En este sentido es importante realizar un análisis de sensibilidad para obtener resultados robustos.

Es pertinente reconocer estas limitaciones, pero aun así es metodológicamente útil y la arbitrariedad en la definición es un precio que debe pagarse para utilizar un concepto que ha probado ser intuitivamente atrayente, socialmente relevante, globalmente aceptado y útil para las discusiones de política (Gasparini et al (2013)).

\subsubsection{Canasta básica}

La Canasta Básica Total (CBT) se utiliza para determinar el nivel de pobreza (moderada) mientras que la Canasta Básica Alimentaria (CBA) para la indigencia (pobreza extrema). La primera se construye a partir de la segunda junto con otros bienes y servicios esenciales. Por lo tanto, para expandir el valor de la CBA se utiliza la inversa del coeficiente de Engels $(\mathrm{CdE})$, definido este último como la relación entre los gastos y alimentos y los gastos totales observados en la población de referencia.

$$
C d E=\frac{\text { Gto alimentos }}{\text { Gto total }}
$$

La estructura del CdE se obtiene de las encuestas de gastos, y para cada período se actualiza por el cambio en los precios relativos de los alimentos respecto a los demás bienes y servicios. De esta forma se actualizan los gastos considerando la misma relación de cantidades consumidas.

$$
C B T=C d E^{-1} * C B A
$$

Las series CBT y la CBA se construyen a partir de las canastas oficiales que publica el INDEC en el año 2016. Cabe mencionar que no se toma la serie desde el año 2003 en adelante, debido a la intervención que sufrió dicha institución a partir del año 2006, generando desconfianza sobre los datos oficiales. En el año 2016, con el cambio de gobierno, se decreta la emergencia estadística ${ }^{2}$, seguida por revisión de la metodología del cálculo de gran parte de sus variables de cartera, entre ellas las que comprenden a la CBT y a la CBA. El nuevo cálculo no involucra un cambio en la metodología, si no que se

\footnotetext{
${ }^{2}$ Decreto 55/2016
} 
actualiza la información que se había utilizado para hacer la canasta anterior, evaluada en el año 1988. Además de actualizar los requerimientos nutricionales, se incorporó una CBA específica para cada región, que difiere de la metodología anterior en la cual se utilizaba la misma canasta corregida por un índice de precios específicos de cada área. Además, se actualiza el coeficiente de Engels. En la primera canasta se utiliza la Encuesta Nacional de Gastos de Hogares del año 1985/86 que luego es reemplazada por la del año 2004/05 (Beccaria 2017).

En este trabajo se construye ambas canastas a partir de la última publicación del 2016 (nueva forma de cálculo) y se construye la serie hacia atrás, tanto para la CBT como para la CBA. Ambas canastas se las deflactan por un índice de precios, que resulta ser un empalme de 8 provincias $^{3}$. Para obtener mayor rigurosidad, se deflactó la CBA sólo por el capítulo que releva la variación de los alimentos, mientras que, para la CBT, se utilizó el índice general que incluye todos los capítulos.

\subsection{Evidencia empírica sobre la dinámica de la pobreza}

\subsubsection{Internacional}

Existen diferentes enfoques que han estudiado la dinámica de la pobreza. Entre los más antiguos se encuentran aquellos que estudian la dinámica de los ingresos entre dos estados: ser pobre o no ser pobre. En este sentido, Lillard y Wills (1998) proponen una metodología econométrica para modelar las ganancias del ciclo de vida y la modalidad entre los estados, utilizando datos de panel para el salario de los hombres que permite estimar una función con componentes permanentes y transitorios seriamente correlacionados, dadas las variables observadas y no observadas. Asumiendo que el error esta normalmente distribuido, desarrollan las pautas para estimar la probabilidad de que los individuos fluctúen entre los diferentes estados. Stevens (1999) y Devincenti (2001) también han empleado este método para el estudio de la pobreza en Estados Unidos y Reino Unido. En estos modelos, se utilizan el ingreso como variable de análisis, lo cual permite aprovechar al máximo el uso de la información, identificando que tan lejos se encuentra el hogar de la

\footnotetext{
${ }^{3} \mathrm{El}$ IPC es una construcción, que agrega diferentes IPC provinciales oficiales. La metodología de este índice se detalla en el anexo.
} 
línea de la pobreza. Por otro lado, en estos modelos no es posible considerar explícitamente los eventos asociados.

Otro de los enfoques, utiliza modelos de Markov de primer orden para el análisis de las transiciones de las personas pobres. Jenkins y Capellari (2002) extienden este enfoque aplicando un modelo probit trivariado, donde además de considerar la probabilidad de ser pobre en el momento inicial y la probabilidad de transitar fuera de la pobreza, estiman la probabilidad de permanecer en el panel, utilizando datos de la década de 1990 del Reino Unido. Su contribución especial radica en que consideran la condición inicial y el problema de attrition ${ }^{4}$ como no aleatorio. Los resultados sugieren que hay un estado de dependencia en la pobreza, y que las condiciones iniciales y los problemas de retención de la muestra son un hecho endógeno en la estimación de las ecuaciones de transición. Si bien este tipo de modelos tienen en cuenta estas variables explicativas, no son tan sofisticados en la dinámica intrínseca de las transiciones, ya que no se tiene en cuenta la duración en el estado de pobreza-no pobreza.

Un tercer enfoque encuadra a los modelos de duración y probabilidad condicional de las transiciones, incorporando en los análisis posibles eventos asociados. En otras palabras, estiman la probabilidad de que suceda un evento y la probabilidad condicional de que el hogar transite fuera y dentro de un estado de pobreza dado que ocurrió dicho evento. Bane y Ellwood (1986) fueron los pioneros en plantear la incorporación de eventos relacionados con el mercado de trabajo y demográficos en el estudio de la dinámica de la pobreza. En tanto, trabajos como el de Jenkins y Schulter (2003) utilizan eventos a través de un modelo probit para analizar el motivo por el cual los ratios de pobreza infantil son mayores en Inglaterra que en Alemania. De los resultados obtienen que las diferencias entre estos países recaen principalmente en las transiciones condicionadas a eventos desencadenantes, más que en la probabilidad de que ocurra el evento en sí. Por lo tanto, concluyen que las diferencias están asociadas a los servicios que provee el Estado, en particular el Estado Alemán provee mejores soluciones para combatir eventos adversos y potenciar los eventos con impacto positivo.

\footnotetext{
${ }^{4}$ Attrition significa desgranamiento en inglés. Desgranamiento de la muestra refiere a la pérdida de observaciones individuales entre dos paneles.
} 
Asimismo, otro tipo de investigaciones utilizan la definición de eventos para identificar cuáles de ellos potencian las tasas de salida de la pobreza. Cantó (2006) analiza el impacto de diferentes eventos en la promoción de la salida de la pobreza para los hogares en España. Mediante un análisis descriptivo y otro multivariado analizan las tasas de salida y el efecto de los eventos, controlando por la composición de los hogares. Entre los resultados, encuentran que las tasas de salida están relacionadas con las características sociodemográfica de los hogares, siendo menor las tasas de salida para hogares con presencia de menores. Además, dentro de estos hogares son los eventos laborales los que tienen mayor incidencia sobre las tasas de salida de la pobreza. De lo contrario, para los hogares que no cuentan con la presencia de menores, son los eventos clasificados como no laborales los que tienen mayor relación con las tasas de salida de la pobreza, explicando que dicha transición está ligada a la protección social del sistema. Sus recomendaciones de política se orientan a mejorar el bienestar de los hogares con presencia de menores para enfrentar los efectos adversos de las fluctuaciones del mercado laboral.

\subsubsection{Argentina}

En la Argentina, hay escasos estudios relacionados con la dinámica de la pobreza. Algunos de ellos se enfocan en un modelo intertemporal, para identificar la pobreza crónica y transitoria. En esta línea, Cruces y Woodon (2003) analizan los determinantes de los componentes crónicos y transitorios a partir de utilizar datos de panel correspondientes a Argentina durante el período 1995-2003. En primer lugar, encuentran que los niveles de pobreza han crecido durante este período y en general los hogares pobres con presencia de menores, presentan peores condiciones laborales y un menor nivel de educación. Diferenciado entre pobreza crónica y transitoria los hogares con jefes de hogares jóvenes experimentan una pobreza más transitoria que crónica. Así también, los que son cuentapropistas también tienen asociada una pobreza más del carácter transitorio que crónico debido a los riesgos asociados a manejar un negocio propio. En otro estudio, Alejo y Garganta (2014) utilizan un modelo tobit para estimar los determinantes de cada componente de la pobreza en Argentina en el período 1997-2012. En base a la Encuesta Permanente de Hogares, construyen paneles que permiten seguir a los hogares en cuatro momentos del tiempo. De aquí surge que el factor transitorio está asociado principalmente 
con las características laborales. En cambio, la estructura demográfica y las cualidades del jefe del hogar inciden sobre el componente crónico.

Un conjunto de estudios, retoma el enfoque de los eventos para caracterizar las transiciones de la pobreza. En esta línea, Beccaria y Maurizio (2006) analizan la dinámica de la pobreza para el período 1991-2003 para el Gran Buenos Aires, identificando un conjunto de eventos diferentes asociados a las tasas de entrada y salida. Dado el contexto del país, tienen en cuenta los efectos adversos que genera la inflación sobre la pobreza. Los resultados sugieren que los eventos relacionados con el mercado de trabajo están más relacionados con las transiciones, tanto por su mayor frecuencia como por su mayor impacto en los ingresos totales familiares. En tanto, los eventos demográficos parecen tener menor relevancia en la dinámica de la pobreza. Entre las recomendaciones de política, los autores sugieren que es prioritario ampliar el paquete de políticas laborales, particularmente a los empleos precarios y a la protección de los trabajadores del tipo cuentapropistas desempleados que no están cubiertos por lo seguros de desempleo. Además, proponen una transferencia monetaria universal no condicionada a hogares con menores que no posean ningún miembro ocupado como asalariado registrado o jubilado, similar a lo que años después fue la Asignación Universal por Hijo. El objetivo es asegurar a los hogares un mínimo nivel de ingreso monetario no vinculado a la situación de sus miembros en el mercado de trabajo, para disminuir las fluctuaciones de los ingresos familiares totales.

Siguiendo la línea temporal Maurizio, Perrot y Villafañe (2008) continúan este análisis, para el período 2003-2007, pero en este caso lo realizan para 28 aglomerados de Argentina, poniendo énfasis en los eventos asociados al mercado de trabajo, transferencias monetarias y eventos demográficos. De la misma manera, encuentran que los eventos relacionados con el mercado del trabajo están más relacionados con las transiciones identificadas, y asimismo observan que los hogares con niños y con jefatura femenina exhiben mayores dificultades para salir de la pobreza. Entre las recomendaciones de política sostienen la importancia que tiene generar un empleo de calidad, conjuntamente con políticas sociales por parte del gobierno nacional que permitan asegurar ciertos niveles de ingresos. En particular advierten que focalizar la política pública en el mercado laboral, tiene alcance para los ocupados y desocupados, pero deja afuera a quienes no forman parte del mercado 
activo y que tampoco están cubiertos por la seguridad social. En esta dirección, plantean como prioritario un conjunto de paquete que incluya una asignación universal a los hogares con menores y una asignación no contributiva a los mayores que no perciban jubilaciones y pensiones.

En el plano internacional, en el estudio de Beccaria et. al. (2012) aplican esta misma metodología para estimar la dinámica de la pobreza en 5 países de Latinoamérica en el período 2003-2008. El objetivo de este trabajo es analizar los ratios de salida y entrada a la pobreza para países con diferentes niveles de pobreza inicial, y poder relacionarlo a diferentes eventos. Entre los resultados encuentran que en general son considerables los casos de transiciones dentro y fuera de la pobreza. Además, encuentran que a pesar de que existe una gran proporción de hogares que experimentaron acontecimientos positivos, principalmente relacionados con el mercado de trabajo, sólo una pequeña fracción de ellos pudo salir de la pobreza. Por otro lado, en general los eventos demográficos y los relacionados con transferencias monetarias resultaron tener poca relevancia. En principio se esperaba que las transferencias monetarias, contribuyan a intensificar las tasas de salida, y de atenuar las tasas de entrada, pero este efecto esperado no pudo ser rectificado por los resultados. En relación a la estructura de los hogares encuentran que aquellos con menores tienen mayor preponderancia a experimentar eventos negativos que los que no poseen. Otro resultado que encuentran es que las transiciones entre un estado de pobreza al de no pobreza ocurre aun cuando a nivel macro la economía se encuentra estable. Los autores concluyen que es causa de la precariedad laboral y de que el sistema de protección social se encuentra poco desarrollado. En un trabajo posterior, Beccaria et. at (2015) realiza el mismo análisis extendiendo el período al año 2012, y los resultados que encuentran son similares.

\section{Fuente de información}

\subsection{Encuesta Permanente de Hogares}

El análisis de la pobreza en una dimensión dinámica requiere la construcción de paneles que permitan realizar un seguimiento de los hogares en el tiempo. Los datos que se utilizarán provienen de la Encuesta Permanente de Hogares Continua (EPHc) entre el 
período 2003 a 2014. En esta encuesta los hogares son encuestados por dos trimestres consecutivos, luego se retiran dos y vuelven a ser entrevistados dos trimestres consecutivos más. Dado el sistema de rotación, se retiene el 50\% de los encuestados, entre el mismo trimestre en dos años consecutivos. En base a esta información, se realizarán paneles anuales. Dentro del período total, el análisis se realiza para tres sub períodos que presentan diferentes periodos de reducción de pobreza: 2003-2006, 2008-2011 y 2011-20145.

En la tabla 3.1 se presenta para cada período el número de hogares en la muestra original, el número de esos hogares con ingresos válidos y finalmente los hogares válidos que resultan de la construcción de paneles. A modo de ejemplo, para el primer período, del total de los hogares el $28 \%$ se los consideran observaciones inválidas por tener ingresos incorrectos o imputados. Luego al armar los paneles, si se realizan los hogares válidos sobre el total se obtiene una pérdida del $78 \%$, que si bien el 50\% se debe a la misma rotación de la muestra la perdida restante se debe al desgranamiento de la muestra o attrition.

La presencia este fenómeno introduce sesgos en la muestra, ya que puede ser pérdida de información no aleatoria. En este trabajo no se corrige este tipo de sesgo debido a que no se obtuvo la información que diferencie la pérdida de datos producto del esquema de rotación de la causada por el desgranamiento de la muestra. Tampoco se contaba con variables que pudieran funcionar como instrumentos válidos en regresiones bivariadas o trivariadas.

En el trabajo que realizan Beccaria y Maurzio (2006) estiman el efecto del desgranamiento sobre la dinámica de la pobreza en Argentina en la década de los noventa encontrando que el mismo no resultó ser muy relevante, y los resultados obtenidos no se modificaron al tener en cuenta este fenómeno.

\footnotetext{
${ }^{5}$ El primero de ellos fue un período de reducción brusca de pobreza. El segundo período, la reducción de la pobreza agregada es moderada, y finalmente el periodo del 2011 al 2014, la reducción de pobreza es menor, incluso se revierte en el último período.
} 
Tabla 3.1 Hogares totales, válidos y pérdida por período

\begin{tabular}{|c|c|c|c|c|}
\hline & & $\begin{array}{l}\text { Período } \\
1\end{array}$ & $\begin{array}{l}\text { Período } \\
2\end{array}$ & $\begin{array}{l}\text { Período } \\
3\end{array}$ \\
\hline \multirow{4}{*}{ Corte transveral } & $\overline{\text { Años }}$ & $\begin{array}{l}2003- \\
2004- \\
2005\end{array}$ & $\begin{array}{l}2008- \\
2009- \\
2010\end{array}$ & $\begin{array}{l}2011- \\
2012- \\
2013- \\
2014\end{array}$ \\
\hline & Hogares totales & 40,790 & 54,519 & 71,583 \\
\hline & Hogares válidos & 29,381 & 39,787 & 50,539 \\
\hline & Primera pérdida & $28.0 \%$ & $27.0 \%$ & $29.4 \%$ \\
\hline \multirow[t]{3}{*}{ Pool paneles } & Años & $\begin{array}{l}2003 / 4- \\
2004 / 5- \\
2005 / 6\end{array}$ & $\begin{array}{l}2008 / 9- \\
2009 / 10- \\
2010 / 11\end{array}$ & $\begin{array}{l}2011 / 12- \\
2012 / 13- \\
2013 / 14\end{array}$ \\
\hline & Hogares válidos & 8,663 & 10,995 & 8,628 \\
\hline & Segunda pérdida & $50.8 \%$ & $52.8 \%$ & $58.5 \%$ \\
\hline Pérdida total & & $78.8 \%$ & $79.8 \%$ & $87.9 \%$ \\
\hline rotación & & $50.0 \%$ & $50.0 \%$ & $50.0 \%$ \\
\hline attrition & & $28.8 \%$ & $29.8 \%$ & $37.9 \%$ \\
\hline
\end{tabular}

Fuente. Elaboración propia. Datos de la Encuesta Permanente de Hogares (EPH-INDEC).

Sin embargo, para tener un control sobre los sesgos que podría tener la muestra dada esta limitación, se realiza una caracterización de los hogares diferenciando entre las muestras de corte transversal y paneles. Las características de ambas muestras son similares. La tabla de características socioeconómicas utilizando datos de panel se encuentra en la tabla 1 de la sección del Anexo.

\section{Enfoque y Metodología}

\subsection{Transiciones}

Una vez definidos los hogares como pobres/indigentes o no pobres/no indigentes, se realizarán las matrices de transición entre cada estado. La tasa de entrada queda definida como los hogares pobres en el segundo período en relación a los no pobres en el primer 
período. De manera inversa, la tasa de salida se define la cantidad de hogares no pobres en el segundo período en relación a los pobres del primer período. Utilizando el método de máxima verosimilitud, se obtiene que estas frecuencias relativas son estimadores consistentes de las probabilidades de transición.

\section{Método de máxima verosimilitud}

La probabilidad de transición se puede expresar como la función de densidad conjunta de la muestra, también definida como la función de máxima verosimilitud.

Para las transiciones de entradas (salidas)

$$
\operatorname{Prob}(\text { Trancisión })=p^{k} p^{n-k}=L(p)
$$

$\mathrm{n}=$ cantidad de hogares no pobres (pobres) en $\mathrm{tl}$

$\mathrm{k}=$ cantidad de hogares pobres (no pobres) en $\mathrm{t} 2$.

Se maximiza la siguiente función asumiendo la restricción de que $0 \leq=p \leq 1$, es decir que se deriva la función y se iguala a cero. Para facilitar el cálculo se puede aplicar logaritmo (ya que el máximo de la función $\mathrm{L}(\mathrm{P})$ coincide con el máximo de la función $\ln (\mathrm{L}(\mathrm{p})$

$$
\begin{gathered}
\ln (L(p))=c t e+k \ln (p)+(n-k) \ln (1-p) \\
\frac{\partial \ln (L(p))}{\partial p}=\frac{k}{p}-\frac{n-k}{1-p} \\
\frac{k}{\hat{p}}-\frac{n-k}{1-\hat{p}}=0 \\
\hat{p}=\frac{k}{n}
\end{gathered}
$$

Se comprueba que es un máximo a partir de la derivada segunda.

$$
\frac{\partial^{2} \ln (L(p))}{\partial^{2} p}=-\frac{k}{p^{2}}-\frac{n-k}{(1-p)^{2}} \leq 0
$$

Debido a que siempre $\mathrm{k} \leq \mathrm{n}$ 
El análisis de las transiciones se realiza en todos los hogares que transitan por la pobreza e indigencia y también diferenciando entre aquellos con y sin presencia de menores.

\subsection{Eventos}

\section{Eventos exhaustivos y excluyentes.}

Una primera alternativa, es realizar una lista exhaustiva de eventos mutuamente excluyentes (Beccaria y Maurizio (2006), Maurizio, Perrot y Villafañe (2008), Beccaria et al (2011)), que permite descomponer las tasas de entradas y salidas a la pobreza como la suma de las transiciones relacionados a todos los eventos identificados.

\section{Eventos simultáneos}

Por otro lado, una segunda alternativa, es definir diferentes eventos que pueden ocurrir de manera simultánea (Jenkins (2003)). Esta alternativa es útil para asociar eventos al estado de pobreza, pero no para descomponer las transiciones.

Una vez definidos los eventos, se caracteriza a las entradas y salidas de la pobreza, identificando la probabilidad de que los eventos se asocien a dichas transiciones.

\section{$\underline{4.3 \text { Descomposición de las probabilidades de transición }}$}

Siguiendo la metodología de Jenkis y Schulter (2001) se estima la distribución de las tasas de entrada y salidas asociadas a dichos eventos. Primero se define a la probabilidad de salir/entrar de la pobreza como la suma de probabilidades de dicha transición asociadas a cada uno de los episodios considerados.

$P\left(S_{i j}\right)=\sum_{r=1}^{R} P\left(S_{i j}, E_{r}\right)$

Donde $S_{i j}$ indica la transición desde el estado de pobreza en " $t$ " al estado de pobreza en " $\mathrm{t}+1$ ". $E_{r}$ Indica la ocurrencia del evento $\mathrm{r}$. Siendo $\mathrm{r}=1,2, \ldots \mathrm{R}$ eventos mutuamente excluyentes.

Esta distribución se puede descomponer en dos factores, por un lado, en la probabilidad de experimentar un determinado evento por parte de los hogares en riesgo, y por otro lado, la 
probabilidad condicional de experimentar una entrada/salida dado que el evento ha sucedido.

Finalmente, con el objetivo de testear la sensibilidad de los resultados, se considerarán sólo aquellas transiciones que ubican a los hogares $10 \%$ por arriba o $10 \%$ por debajo de la línea.

\section{$\underline{\text { Eventos }}$}

Con el fin de descomponer las tasas de salida, se generan eventos de acuerdo a la primera alternativa, episodios del carácter exhaustivos y excluyentes:

- Eventos laborales (YL)

- Variación en la cantidad de empleados dentro del hogar

- Variación el ingreso horario de los que están ocupados

- Variación en la cantidad de horas de los que están ocupados

- Variación en el ingreso horario y horas de los que están ocupados

- Variación en el ingreso laboral y cantidad de empleados

- Eventos no laborales (YNL)

- Variación en el ingreso por jubilación

- Variación en ingresos por políticas de transferencias

- Variación de otros ingresos

- Evento laboral y no laboral (YL + YNL)

- Variación en el ingreso laboral y en de transferencias

- Variación en el ingreso laboral y en de otros ingresos laborales

- Variación en ingreso laboral y en anteriores

- Demográfico puro

○ Demográfico + ingreso (YL + YNL)

- Variación de miembro y en el ingreso Total Familiar

- Sin clasificar 


\section{Evolución de pobreza e indigencia $\mathrm{v}$ perfiles de los jefes de}

\section{hogares}

\subsection{Pobreza}

En esta sección realiza un análisis descriptivo de la evolución agregada de la pobreza. Luego de la devaluación que el país experimenta en el año 2001, los niveles de pobreza tomaron sus valores máximos. Según datos de la Encuesta Permanente de Hogares, para el segundo semestre del $2003^{6}$ el $60,3 \%$ de las personas se encontraban por debajo de la línea de la pobreza ${ }^{7}$, consecuencia del aumento de los precios, junto con un aumento del desempleo. Luego de la profunda crisis macroeconómica y el cambio de régimen, comenzó la recuperación con una marcada fase de crecimiento que permitió bajar los niveles de pobreza. En la figura 5.1 se observa cómo fueron disminuyendo la cantidad de pobres durante la fase expansión.

Durante el período 2003- 2006 la pobreza se redujo en aproximadamente 16 puntos porcentuales (p.p.). Estos tres años se experimentó la mayor reducción (con pendiente negativa) En el 2002 se produce un pico de pobreza (pautando el punto de partida de la serie en el 2003 es relativamente alto). Esto sucedió, en parte por la entrada a la pobreza de aquellos hogares con ingresos próximos a la línea de la pobreza luego de sufrir una abrupta disminución en sus ingresos nominales. Con la recuperación económica de los años posteriores, aquellos que habían caído por la crisis se logran recuperar, motivo por el cual la reducción fue la mayor en tan sólo un período de tres años.

Posteriormente, continúa la tendencia de la reducción de la pobreza hasta el año 2013, donde la misma alcanza alrededor del $31 \%$ de las personas. Por lo tanto, se redujo alrededor de 13 p.p. en 7 años. Finalmente, los últimos dos años de análisis (2014 - 2015) los niveles de pobreza aumentan levemente en el 2014 y en último año la evidencia revela que alrededor del $33 \%$ de las personas se encuentran en situación de pobreza en Argentina en este período.

\footnotetext{
${ }^{6} \mathrm{EPH}$ continua se obtiene a partir del 2do trimestre del 2003

${ }^{7}$ Según el método de la Canasta Básica Total
} 
Analizando la pobreza por hogar se observa que en el 2004 el 47\% de los hogares son pobres, y la reducción de la pobreza es menor durante el primer período, alcanzando el $31 \%$ en el año 2007 (caída de 16 p.p.). Luego en el 2013 la pobreza por hogar se ubica en torno al $22 \%$ y termina el período en $23 \%$. La diferencia en las proporciones entre personas y hogares pobres, sugiere que los hogares pobres son más numerosos en cantidad de miembros que los hogares no pobres. Por tanto, las características demográficas que determinan el tamaño del hogar son un punto relevante en la incidencia de la pobreza.

Figura 5.1. Hogares y población pobre (\%). Período 2003 a 2015

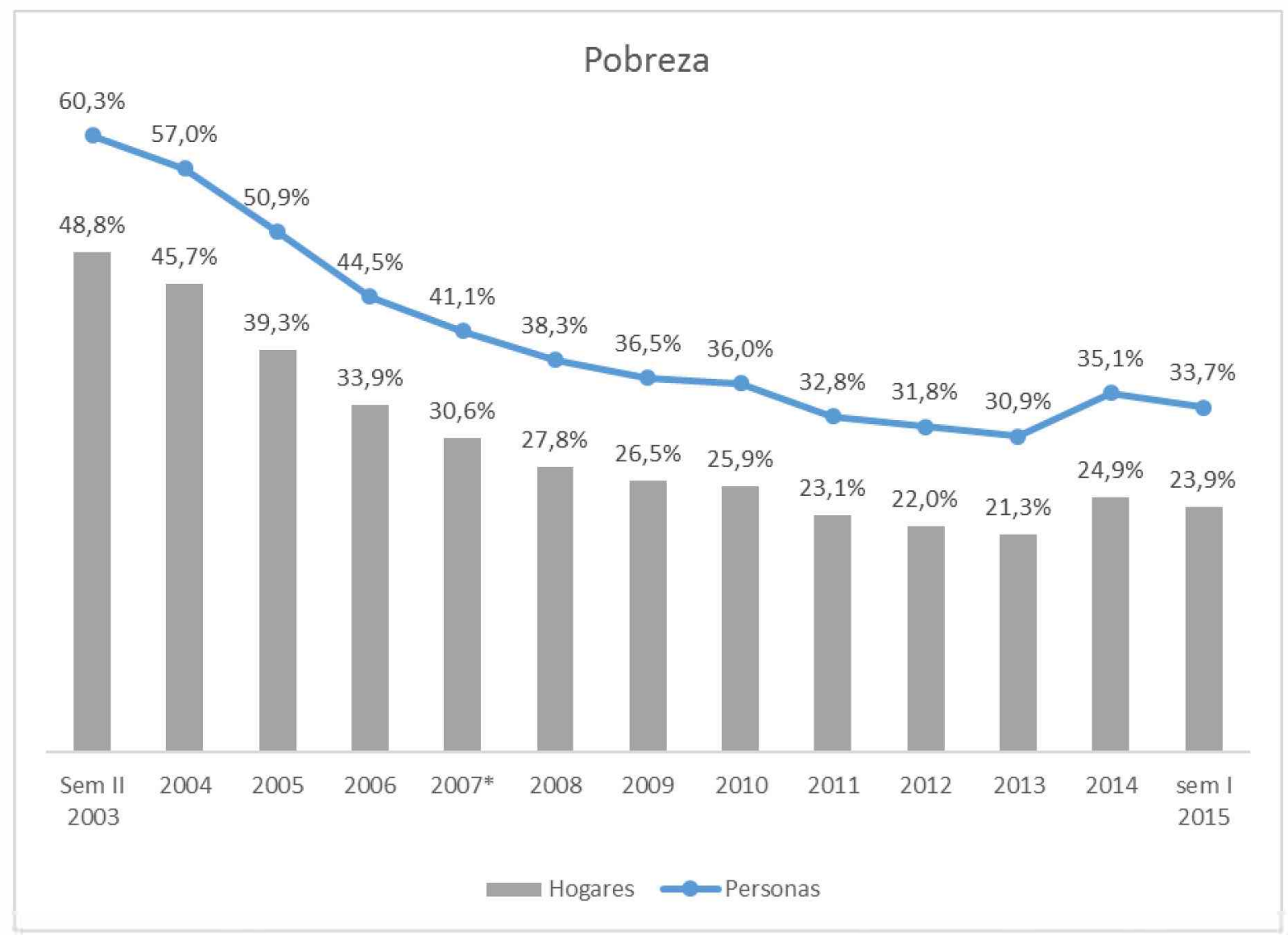

Fuente. Elaboración propia. Datos de la Encuesta Permanente de Hogares (EPH-INDEC).

*No se cuentan con datos pertenecientes al tercer trimestre del año 2007.

\subsection{Indigencia}

Respecto a la pobreza extrema, se observa que al igual que la pobreza moderada, en el año 2003 se registran los mayores niveles de indigencia (Figura 5.2). El 17\% de las personas son consideradas indigentes, mientras que en hogares este porcentaje es de $13 \%$. La 
dinámica de la indigencia revela un descenso más pronunciado en los primeros años de recuperación luego de la crisis, pero a un ritmo menor sigue disminuyendo en los años posteriores hasta el año 2013, tomando valores de 6\% en personas y 5\% en hogares. Similar a la trayectoria de la pobreza en el 2014 hay un aumento, pero vuelve a los mismos valores en el año 2015.

Figura 5.2 Hogares y Personas Indigentes (\%). Período 2003-2015

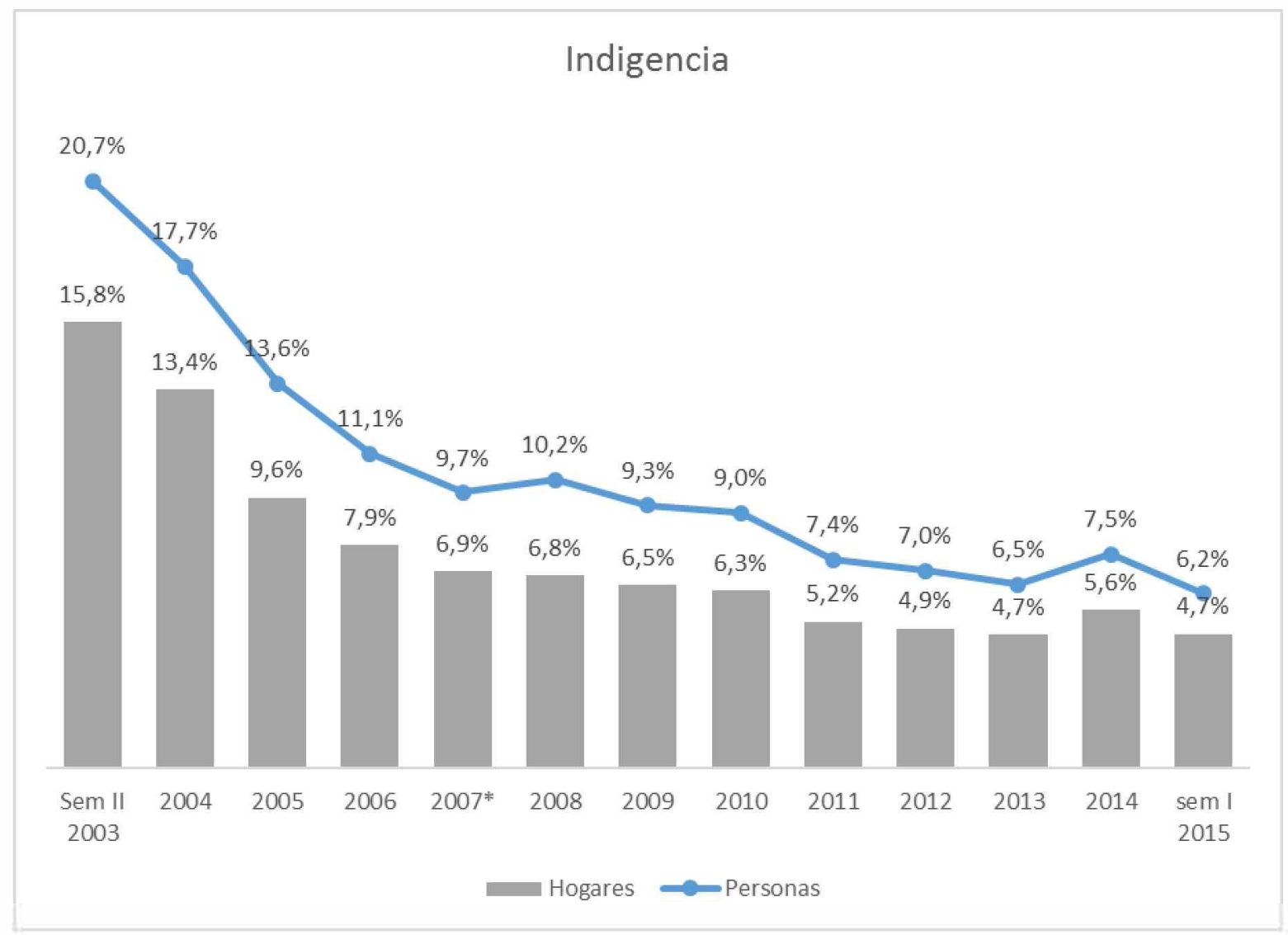

Fuente: Elaboración propia. Datos de la Encuesta Permanente de Hogares (EPH-INDEC).

*No se cuentan con datos pertenecientes al tercer trimestre del año 2007.

\subsection{Características de los hogares}

Parte de este análisis es caracterizar el perfil de la población caracterizada como pobre o no pobre. En la Tabla 5.1, se presentan las características de los jefes de hogar, tales como condiciones educativas, edad, sexo, categoría ocupacional y composición del hogar, según su condición de pobreza. La primera columna explica la composición total de los hogares dentro de cada característica analizada, por ejemplo, el $54.6 \%$ de los hogares tienen jefe de 
hogar con educación inferior o igual a primaria completa. Las otras dos columnas indican la proporción de pobres y de no pobres por categoría de la condición, en este caso el $40,4 \%$ de los hogares con jefe que tiene hasta primaria completa están bajo la línea de la pobreza.

Como era de esperar, los niveles más bajos de educación de los jefes se asocian con mayor incidencia de la pobreza, ya que dentro de cada categoría la proporción de pobres es mayor a menos nivel de instrucción. En relación a la edad del jefe de hogar, la mayoría de los se concentran entre los 25 y 65 años. De estos jefes de hogares aproximadamente el 33\% son pobres. Para los hogares con jefes mayores a 65 años, se observa que el porcentaje de pobres disminuye a la mitad $(14,5 \%)$.

Al mando de los hogares predominan los jefes hombres ya que el $65,4 \%$ de los hogares tiene jefe masculino y el 34,6\% jefe femenino. La evidencia sugiere proporciones similares de hogares pobres dentro de cada categoría. A nivel laboral el 30,2\% de los jefes de hogares se declaran inactivos, probablemente la mayoría por estar retirados, mientras que el $33,2 \%$ declaran ser asalariados formales y 16,2\% trabajan en el sector informal. Dentro de esta última categoría, casi el 50\% de los hogares se encuentra debajo de la línea de la pobreza.

Es importante notar que para aquellos hogares que tienen jefe de hogar desocupado, el 68\% es pobre y el $32 \%$ no lo es. Hay varias cuestiones para detenerse, en primer lugar, que el jefe esté desocupado no se asocia directamente a pobreza del hogar, que se explica a que pueden estar percibiendo ingresos laborales de otros miembros, por ejemplo, ingreso de un conyugue, o también ingresos no laborales, que pueden ser del tipo de becas, subsidios gubernamentales, rentas, etc.

Una de las condiciones más relevantes al momento de caracterizar la pobreza a nivel del hogar, es su composición. De esta muestra se observa que la presencia de menores es un factor que se asocia con mayores niveles de pobreza. Del total de hogares, $47,5 \%$ cuenta con la presencia de menores, de los cuales el $45,2 \%$ de ellos son pobres. 
Tabla 5.1 Característica de los jefes de hogares según condición de pobreza (2003-2014)

\begin{tabular}{|c|c|c|c|c|}
\hline & \multicolumn{4}{|c|}{ Corte Transversal } \\
\hline & $\begin{array}{c}\text { Comp. } \\
\text { total }\end{array}$ & $\begin{array}{c}\text { No } \\
\text { pobres }\end{array}$ & Pobres & Total \\
\hline \multicolumn{5}{|l|}{ Niveles educativos } \\
\hline Hasta primaria completa & $54.6 \%$ & $59.6 \%$ & $40.4 \%$ & $100.0 \%$ \\
\hline Secundaria Incompleta y completa & $30.8 \%$ & $80.1 \%$ & $19.9 \%$ & $100.0 \%$ \\
\hline Universitaria Incompleta y completa & $14.6 \%$ & $93.8 \%$ & $6.2 \%$ & $100.0 \%$ \\
\hline Total & $100.0 \%$ & $70.9 \%$ & $29.1 \%$ & $100.0 \%$ \\
\hline \multicolumn{5}{|l|}{ Edad } \\
\hline Edad $<25$ & $4.4 \%$ & $63.6 \%$ & $36.4 \%$ & $100.0 \%$ \\
\hline $25<=\mathrm{Edad}<=40$ & $30.6 \%$ & $65.4 \%$ & $34.6 \%$ & $100.0 \%$ \\
\hline $41<=\operatorname{Edad}<=65$ & $43.1 \%$ & $68.1 \%$ & $31.9 \%$ & $100.0 \%$ \\
\hline Edad $>65$ & $21.8 \%$ & $85.5 \%$ & $14.5 \%$ & $100.0 \%$ \\
\hline Total & $100.0 \%$ & $70.9 \%$ & $29.1 \%$ & $100.0 \%$ \\
\hline \multicolumn{5}{|l|}{ Sexo } \\
\hline Mujer & $34.6 \%$ & $72.5 \%$ & $27.5 \%$ & $100.0 \%$ \\
\hline Hombre & $65.4 \%$ & $70.0 \%$ & $30.0 \%$ & $100.0 \%$ \\
\hline Total & $100.0 \%$ & $70.9 \%$ & $29.1 \%$ & $100.0 \%$ \\
\hline \multicolumn{5}{|l|}{ Categoría ocupacional } \\
\hline Asalariados registrados & $33.2 \%$ & $84.2 \%$ & $15.8 \%$ & $100.0 \%$ \\
\hline Asalariados no registrados & $16.2 \%$ & $51.0 \%$ & $49.0 \%$ & $100.0 \%$ \\
\hline Cuentapropistas & $13.5 \%$ & $60.1 \%$ & $39.9 \%$ & $100.0 \%$ \\
\hline Patrones & $2.9 \%$ & $85.9 \%$ & $14.1 \%$ & $100.0 \%$ \\
\hline Desocupados & $3.9 \%$ & $32.0 \%$ & $68.0 \%$ & $100.0 \%$ \\
\hline Inactivos & $30.2 \%$ & $75.3 \%$ & $24.7 \%$ & $100.0 \%$ \\
\hline Total & $100.0 \%$ & $70.9 \%$ & $29.1 \%$ & $100.0 \%$ \\
\hline \multicolumn{5}{|l|}{ Composición del Hogar } \\
\hline Hogares unipersonales $=>65$ & $8.7 \%$ & $93.8 \%$ & $6.2 \%$ & $100.0 \%$ \\
\hline Hogares unipersonales $=<65$ & $12.0 \%$ & $87.5 \%$ & $12.5 \%$ & $100.0 \%$ \\
\hline Hogares compuestos jefe $=>65$ sin menores & $10.9 \%$ & $84.9 \%$ & $15.1 \%$ & $100.0 \%$ \\
\hline Hogares compuestos jefe $=<65$ sin menores & $20.8 \%$ & $81.1 \%$ & $18.9 \%$ & $100.0 \%$ \\
\hline Hogares compuestos con menores & $47.5 \%$ & $54.8 \%$ & $45.2 \%$ & $100.0 \%$ \\
\hline Total & $100.0 \%$ & $70.9 \%$ & $29.1 \%$ & $100.0 \%$ \\
\hline Hogares con menores & $47.5 \%$ & $54.8 \%$ & $45.2 \%$ & \\
\hline
\end{tabular}

Fuente. Elaboración propia. Datos de la Encuesta Permanente de Hogares (EPH-INDEC). 
En general la disminución de la pobreza no ha sido homogénea para todos los hogares, y ha sido más intensa para hogares con ciertas características. La tabla 5.2 expone las características de los hogares en el año 2003 y en el año 2014, descomponiendo por condición de pobreza. Los hogares sin presencia de menores obtienen un mayor descenso en los niveles de pobreza, que los hogares con menores. En el 2003 el 67,4\% de los pobres tienen menores a cargo, mientras que en el 2014 la participación aumenta al 80\%, debido a la mayor salida de hogares sin menores relativamente ${ }^{8}$.

Tabla 5.2 Evolución composición del hogar, según condición de pobreza

\begin{tabular}{|c|c|c|c|c|c|c|}
\hline & & 2003 & & & 2014 & \\
\hline Composición del Hogar & $\begin{array}{c}\text { No } \\
\text { pobres }\end{array}$ & Pobres & Total & $\begin{array}{c}\text { No } \\
\text { pobres }\end{array}$ & Pobres & Total \\
\hline Hogares unipersonales $=>65$ & $82.4 \%$ & $17.6 \%$ & $100.0 \%$ & $97.5 \%$ & $2.5 \%$ & $100.0 \%$ \\
\hline Hogares unipersonales $=<65$ & $72.9 \%$ & $27.1 \%$ & $100.0 \%$ & $90.3 \%$ & $9.7 \%$ & $100.0 \%$ \\
\hline Hogares compuestos jefe $=>65$ sin menores & $64.7 \%$ & $35.3 \%$ & $100.0 \%$ & $94.4 \%$ & $5.6 \%$ & $100.0 \%$ \\
\hline Hogares compuestos jefe $=<65$ sin menores & $63.1 \%$ & $36.9 \%$ & $100.0 \%$ & $84.6 \%$ & $15.4 \%$ & $100.0 \%$ \\
\hline Hogares compuestos con menores & $33.8 \%$ & $66.2 \%$ & $100.0 \%$ & $58.5 \%$ & $41.5 \%$ & $100.0 \%$ \\
\hline Total & $51.7 \%$ & $48.3 \%$ & $100.0 \%$ & $74.9 \%$ & $25.1 \%$ & $100.0 \%$ \\
\hline Hogares unipersonales $=>65$ & $15.6 \%$ & $3.6 \%$ & $9.8 \%$ & $12.0 \%$ & $0.9 \%$ & $9.2 \%$ \\
\hline Hogares unipersonales $=<65$ & $13.5 \%$ & $5.4 \%$ & $9.6 \%$ & $15.1 \%$ & $4.9 \%$ & $12.6 \%$ \\
\hline Hogares compuestos jefe $=>65$ sin menores & $13.6 \%$ & $8.0 \%$ & $10.9 \%$ & $13.2 \%$ & $2.3 \%$ & $10.5 \%$ \\
\hline Hogares compuestos jefe $=<65$ sin menores & $25.1 \%$ & $15.7 \%$ & $20.6 \%$ & $21.9 \%$ & $11.9 \%$ & $19.4 \%$ \\
\hline Hogares compuestos con menores & $32.2 \%$ & $67.4 \%$ & $49.2 \%$ & $37.8 \%$ & $80.0 \%$ & $48.4 \%$ \\
\hline Total & $100.0 \%$ & $100.0 \%$ & $100.0 \%$ & $100.0 \%$ & $100.0 \%$ & $100.0 \%$ \\
\hline
\end{tabular}

Fuente. Elaboración propia. Datos de la Encuesta Permanente de Hogares (EPH-INDEC).

Los resultados presentados hasta aquí, se analizan desde un punto de vista estático, en la siguiente sección se analizan las transiciones adoptando un enfoque dinámico.

\footnotetext{
${ }^{8}$ Los datos presentados en esta sección corresponden a la sección cruzada, sin embargo, los resultados son similares cuando se analizan el pool de paneles.
} 


\section{Resultados dinámicos}

\subsection{Tasas de entrada y salida de la pobreza e indigencia}

\section{Pobreza}

Para el conjunto de períodos analizados ${ }^{9}$, las transiciones entre dos observaciones entre las cuales media un año se exponen en la tabla 6.1. Las tasas de entradas corresponden entonces, a aquellos hogares que son "no pobres" en la primera observación y que son "pobres" en la segunda observación. De manera opuesta, las tasas de salida equivalen a los hogares que clasificaron como "pobres" en la primera observación y como "no pobres" en la segunda. Este resultado sugiere que la probabilidad de que un hogar inicialmente no pobre caiga en la pobreza es en promedio un año es $9,8 \%$, mientras que la probabilidad de salir de dicho estado es en promedio de $29,3 \%$ para el total de períodos.

Tabla 6.1 Tasas de entrada (a) y de salida (de) la pobreza, según composición del hogar

\begin{tabular}{|c|c|c|c|c|c|c|}
\hline \multirow{2}{*}{ 2003-2014 } & \multicolumn{2}{|c|}{ Total } & \multirow{2}{*}{\multicolumn{2}{|c|}{$\begin{array}{c}\text { Con hijos } \\
\text { Estado en 2da observación }\end{array}$}} & \multicolumn{2}{|c|}{ Sin hijos } \\
\hline & & & & & & \\
\hline Estado en 1ra observación & Pobres & No pobres & Pobres & No pobres & Pobres & No pobres \\
\hline Pobres & $70,7 \%$ & $29,3 \%$ & $77,5 \%$ & $22,5 \%$ & $52,3 \%$ & $47,7 \%$ \\
\hline No pobres & $9,8 \%$ & $90,2 \%$ & $16,1 \%$ & $83,9 \%$ & $6.4 \%$ & $93,6 \%$ \\
\hline
\end{tabular}

Fuente. Elaboración propia. Datos de la Encuesta Permanente de Hogares (EPH-INDEC).

Controlando por la composición del hogar, es notable la brecha que existe entre hogares con y sin presencia de menores, siendo más vulnerables los primeros, con mayores tasas de entrada y menores de salida. Específicamente, la tasa de salida de los hogares sin presencia de menores es el doble que la de los hogares con menores, ocurriendo lo opuesto en las tasas de entrada, ya que las tasas del segundo grupo son 2,5 veces las tasas del primero.

\section{Indigencia}

La tabla 6.2 expone las tasas de entrada y salida de los hogares para este grupo de población. Las tasas de entrada a la indigencia para la década en total fueron en promedio de $3,2 \%$ mientras que la de salida $54,6 \%$.

\footnotetext{
${ }^{9}$ Incluye los paneles entre el 2003 al 2014, excepto por los paneles 2006-2007 y 2007-2008 por no estar disponible el año 2007.
} 
Tabla 6.2 Tasas de entrada (a) y de salida (de) la indigencia, según composición del hogar

\begin{tabular}{ccccccc}
\hline & \multicolumn{2}{c}{ Total } & \multicolumn{2}{c}{$\begin{array}{c}\text { Con hijos } \\
\text { 2003-2014 }\end{array}$} & \multicolumn{3}{c}{ Sin hijos } \\
\hline Estado en 2da observación & \\
Indigentes & Indigentes & No Indigentes & Indigentes & No Indigentes & Indigentes & No Indigentes \\
No Indigentes & $45,4 \%$ & $54,6 \%$ & $48,3 \%$ & $51,7 \%$ & $35,2 \%$ & $64,8 \%$ \\
\hline
\end{tabular}

Fuente. Elaboración propia. Datos de la Encuesta Permanente de Hogares (EPH-INDEC).

Desagregando por composición del hogar, al igual que en la pobreza, la brecha de las entradas es de más de 2,5, sin embargo, se observa que la brecha entre las tasas de salida de la indigencia es menor que en la pobreza, ya que la tasa de salida para los hogares sin menores es 1,2 veces la de los hogares sin menores (en la pobreza esta tasa equivale a más de 2 veces).

\subsection{Eventos asociados a las tasas de salida}

Si bien es importante entender ambos tipos de transiciones, los hogares que entraron a la pobreza en cada año se han mantenido relativamente constantes en promedio (figura 6.1). En cambio, se observa que la dinámica de la reducción de la pobreza está más relacionada con hogares que salen. Por esta razón, este trabajo se focaliza sobre los hogares que experimentaron eventos que les ha permitido salir de la pobreza y de la indigencia. 
Figura 6.1. Tasas de entrada y salida de la pobreza, paneles anuales.
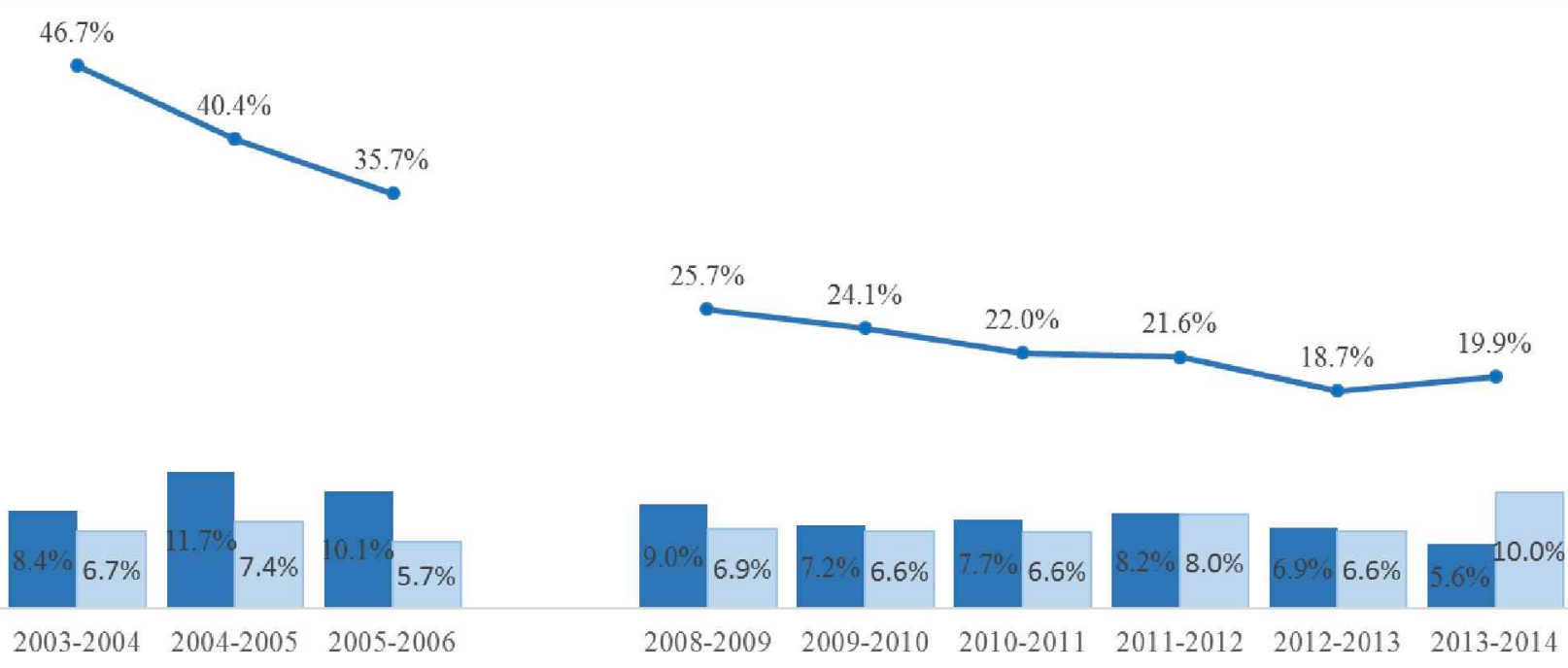

SALIERON/TOTAL $\square$ ENTRARON/TOTAL $=$ POBRE

Fuente. Elaboración propia. Datos de la Encuesta Permanente de Hogares (EPH-INDEC).

\section{Pobreza}

\section{$\underline{\text { Período total }}$}

La tabla 6.3 se expresan los resultados para el total de los hogares inicialmente pobres. La tercera columna $(\mathrm{P}(\mathrm{S}))$ indica la tasa de salida asociada a cada episodio considerado. En la primera y segunda columna se desagrega esta probabilidad, siguiendo la ecuación [1] de la sección metodológica, en la frecuencia de cada uno de los eventos (columna 1, P(E)) y en la probabilidad condicional de que el hogar efectivamente salga de la pobreza dado que el evento ocurrió (columna 2, $\mathrm{P}(\mathrm{E} / \mathrm{S})$ ). La probabilidad total expuesta en la tercera columna es, entonces, el producto de las primeras dos.

El primer resultado a destacar es que el $80 \%$ de los hogares experimentó algún evento que potencialmente le permite salir de la situación de la pobreza. Sin embargo, sólo el 29,3\% logra salir efectivamente de este estado. Distinguiendo por hogar se observa que la probabilidad de ocurrencia para el conjunto de episodios es similar $(79,7 \%$ y $81,3 \%$ para 
hogares con y sin presencia de menores respectivamente), pero tal como se mencionó en apartados anteriores difieren en la probabilidad total, consecuencia de la brecha en la probabilidad condicional.

El segundo resultado a destacar es que los eventos laborales son los más importantes para ambos tipos de hogares, tal como se indica el trabajo de Maurizio, Perrot y Villafañe (2008) hay evidencia de que gran parte de las transiciones se explican a través del mercado de trabajo, y por lo tanto mejorar las condiciones laborales para asegurar mejores ingresos es una condición necesaria para avanzar con la reducción de los niveles de pobreza.

La tasa de salida total (columna 3) para los hogares que han experimentado la entrada de un trabajador al mercado laboral informal es mayor en promedio que en los hogares que han experimentado la entrada de un trabajador formal. Si bien la diferencia es pequeña, esta se debe a la mayor probabilidad de ocurrencia de obtener un trabajo informal. Analizando por hogar, se observa que la probabilidad total se revierte para los hogares con presencia de menores, ya que, si bien la probabilidad de ocurrencia de obtener un empleo informal sigue siendo mayor, la probabilidad total de que salga dado que obtuvo el empleo es mayor en el mercado formal $(0.4 \%$ formal vs $0.2 \%$ informal $)$, producto de la brecha de ingresos entre ambos tipos de contratación laboral.

Igualmente, dentro de los eventos laborales, los que se asocian con mayores probabilidades de salida, son aquellos que combinan aumentos en ingreso horario y en la cantidad de horas. Este resultado se mantiene al interior de los grupos analizados (alrededor del 3\%). Sin embargo, vuelve a suceder que la probabilidad de ocurrencia es mayor para los hogares con presencia de menores pero menor su probabilidad condicional en relación a los hogares sin presencia de estos. Este resultado se repite a lo largo del trabajo.

Los eventos demográficos puro tienen baja relación con las tasas de salida en ambos tipos de hogares, en parte porque la probabilidad de que ocurra el evento por sí sólo es baja.

La relevancia de los eventos asociados a ingresos no laborales puros es diferente para cada tipo de hogar. Para los hogares con presencia de menores la probabilidad total es baja sólo del $0,5 \%$, mientras que en los hogares sin menores es del 13,2\%. Esta brecha se explica principalmente por los ingresos por jubilación. 
El efecto de los ingresos no laborales puro en los hogares con presencia de menores resulta bajo, principalmente porque es poco probable que el hogar sólo reciba ingresos no laborales, dado que en la mayoría de los casos hay algún tipo de ingreso adicional proveniente del mercado de trabajo. Además, en los pocos casos donde la única fuente de ingresos es no laboral, la probabilidad condicional de salida es demasiado baja, dando cuenta de que no son ingresos suficientes para salir del estado. 
Tabla 6.3 Descomposición de las tasas de salida de la pobreza según eventos. Período 2003-2014

\begin{tabular}{|c|c|c|c|c|c|c|c|c|c|}
\hline \multirow[b]{2}{*}{ Eventos } & \multicolumn{3}{|c|}{ Total } & \multicolumn{3}{|c|}{ Con menores } & \multicolumn{3}{|c|}{ Sin menores } \\
\hline & $\mathrm{P}(\mathrm{E})$ & $\mathrm{P}(\mathrm{S} / \mathrm{E})$ & $\mathrm{P}(\mathrm{S})$ & $\mathrm{P}(\mathrm{E})$ & $\mathrm{P}(\mathrm{S} / \mathrm{E})$ & $\mathrm{P}(\mathrm{S})$ & $\mathrm{P}(\mathrm{E})$ & $\mathrm{P}(\mathrm{S} / \mathrm{E})$ & $\mathrm{P}(\mathrm{S})$ \\
\hline 1.Eventos laborales & 31.9 & 35.6 & 11.3 & 35.3 & 29.9 & 10.5 & 22.7 & 59.2 & 13.5 \\
\hline 1.1.1 Aumento de cantidad de asalariados registrados sin entrada de un miembro ocupado al hogar & 1.0 & 44.9 & 0.4 & 1.1 & 35.3 & 0.4 & 0.5 & 100.0 & 0.5 \\
\hline 1.1.2 Aumento de cantidad de asalariados no registrados sin entrada de un miembro ocupado al hogar & 2.4 & 25.5 & 0.6 & 2.2 & 10.2 & 0.2 & 2.9 & 56.6 & 1.6 \\
\hline 1.1.3 Aumento de cantidad de no asalariados sin entrada de un miembro ocupado al hogar & 1.1 & 25.0 & 0.3 & 0.4 & 7.3 & 0.0 & 2.7 & 32.9 & 0.9 \\
\hline 1.2 Aumento en el ingreso horario de quienes están siempre ocupados & 11.5 & 34.6 & 4.0 & 13.2 & 29.4 & 3.9 & 6.7 & 62.4 & 4.2 \\
\hline 1.3 Aumento en la cantidad de horas trabajadas de los que están siempre ocupados & 3.2 & 13.7 & 0.4 & 3.7 & 9.2 & 0.3 & 1.9 & 37.4 & 0.7 \\
\hline 1.4 Aumento en el ingreso horario y en la cantidad de horas trabajadas (combinación 1.2 y 1.3 ) & 7.6 & 38.6 & 3.0 & 8.8 & 33.9 & 3.0 & 4.5 & 63.5 & 2.9 \\
\hline $\begin{array}{l}\text { 1.5 Aumento en la cantidad de ocupados y en el ingreso laboral de quienes están siempre ocupados } \\
\text { (combinación de todos los anteriores) }\end{array}$ & 5.1 & 52.0 & 2.6 & 5.7 & 46.6 & 2.7 & 3.4 & 76.4 & 2.6 \\
\hline 2. Eventos No Laborales & 11.3 & 34.7 & 3.9 & 5.8 & 8.1 & 0.5 & 26.1 & 50.5 & 13.2 \\
\hline 2.1 Aumento de ingresos por jubilación & 6.6 & 44.0 & 2.9 & 2.0 & 13.4 & 0.3 & 19.1 & 52.7 & 10.1 \\
\hline 2.2 Aumento de ingresos por politicas de transferencias & 2.2 & 1.9 & 0.0 & 2.7 & 1.0 & 0.0 & 0.9 & 8.8 & 0.1 \\
\hline 2.3 Aumento de otros ingresos no laborales (resto-jub-trans) & 2.4 & 38.8 & 0.9 & 1.1 & 16.1 & 0.2 & 6.1 & 49.5 & 3.0 \\
\hline 3. Aumento de ingreso laboral y aumento de trans ferencias (ingreso no laboral) & 9.0 & 27.5 & 2.5 & 11.7 & 27.2 & 3.2 & 1.6 & 34.3 & 0.6 \\
\hline 4. Aumento de ingreso laboral y aumento de otros ingresos no laborales (no transferencias) & 7.0 & 59.3 & 4.1 & 5.7 & 43.7 & 2.5 & 10.5 & 82.1 & 8.6 \\
\hline 5. Combinación de aumento en ingresos laborales y aumentos de ambos anteriores & 1.4 & 27.2 & 0.4 & 1.9 & 24.7 & 0.5 & 0.3 & 70.0 & 0.2 \\
\hline 6. Demográfico puro (reducción en la cantidad de miembros sin aumento del ingreso total familiar) & 5.4 & 16.4 & 0.9 & 5.6 & 14.7 & 0.8 & 4.7 & 21.9 & 1.0 \\
\hline 7. Aumento en la cantidad ocupados & 0.9 & 20.8 & 0.2 & 0.9 & 15.1 & 0.1 & 1.0 & 34.4 & 0.3 \\
\hline 8. Reducción miembro + aumento en el ingreso total, incluido aumento de transferencias & 1.1 & 42.3 & 0.5 & 1.4 & 39.9 & 0.6 & 0.3 & 77.0 & 0.2 \\
\hline 9. Reducción miembro + aumento en el ingreso total, sin aumento de transferencias & 5.2 & 60.6 & 3.2 & 4.9 & 47.1 & 2.3 & 6.3 & 88.7 & 5.6 \\
\hline 10. Hogares sin clasificar & 7.0 & 34.1 & 2.4 & 6.7 & 23.7 & 1.6 & 7.7 & 58.5 & 4.5 \\
\hline Eventos con aumento de trans férencias & 14.6 & 24.3 & 3.5 & 18.5 & 23.5 & 4.3 & 4.1 & 34.2 & 1.4 \\
\hline Hogares con Eventos (\%) & 80.1 & 36.6 & 29.3 & 79.7 & 28.3 & 22.5 & 81.3 & 58.7 & 47.7 \\
\hline Hogares sin eventos $(\%)$ & 19.9 & & & 20.3 & & & 18.7 & & \\
\hline Hogares totales $(\%)$ & 100.0 & & & 100.0 & & & 100.0 & & \\
\hline
\end{tabular}

Fuente. Elaboración propia. Datos de la Encuesta Permanente de Hogares (EPH-INDEC). 
Con el objetivo de indagar en mayor detalle sobre los eventos asociados a transferencias desde el Estado, se realiza la sumatoria de todos los eventos que incluyen este tipo de transferencias, siendo metodológicamente válido, por ser eventos mutuamente excluyentes.

La Tabla 6.4 muestra que eventos fueron sumados para obtener el conjunto de eventos con transferencias.

Tabla 6.4. Eventos que involucran transferencias

\begin{tabular}{l}
\hline Eventos \\
\hline Aumento transferencias \\
Aumento ingreso laboral y sólo transferencias \\
Aumento ingreso laboral, transferencias y otros ingresos no laborales \\
Demográficos y aumento que contiene transferencias \\
Demográfico y sólo transferencias \\
\hline Total de eventos con transferencias \\
\hline
\end{tabular}

La probabilidad total de que un hogar salga asociado a un evento de transferencias es de $3,5 \%$ para el total de hogares. Dicho de otra forma, el $12 \%$ de las tasas de salida se asocia con transferencias (Tabla 6.3).

Al comparar al interior de cada hogar, las transferencias del Estado toman mayor importancia entre los hogares con menores. La tasa total de salida asociada es de 4,1\%, (equivalente a un 19\% de las tasas de salida de estos hogares), mientras que, para los hogares sin menores, representan sólo el 3\%.

Es interesante notar que este número se debe a una mayor probabilidad de ocurrencia de los eventos en los hogares con menores. En una primera aproximación, es posible resaltar que si bien las transferencias parecen estar enfocadas en este tipo de hogares la probabilidad de que un hogar salga dado que recibió las transferencias es en promedio alrededor del $30 \%$, en cambio para los hogares con menores su probabilidad condicional es en promedio aproximadamente el doble. 


\section{$\underline{\text { Sub períodos }}$}

La figura 6.2 expone las tasas de salidas asociadas a conjuntos de episodios a lo largo del tiempo. Como primer resultado resulta que la tasa de salida sólo por evento laboral se ha mantenido en promedio relativamente constante, mientras que, sí se observa un aumento en los ingresos no laboral, reflejado en el aumento de la participación de este evento en las tasas de salida, como así también en combinación con los eventos laborales.

Figura 6.2 Tasas de salida total, según composición del hogar y tipo de evento. Sub períodos

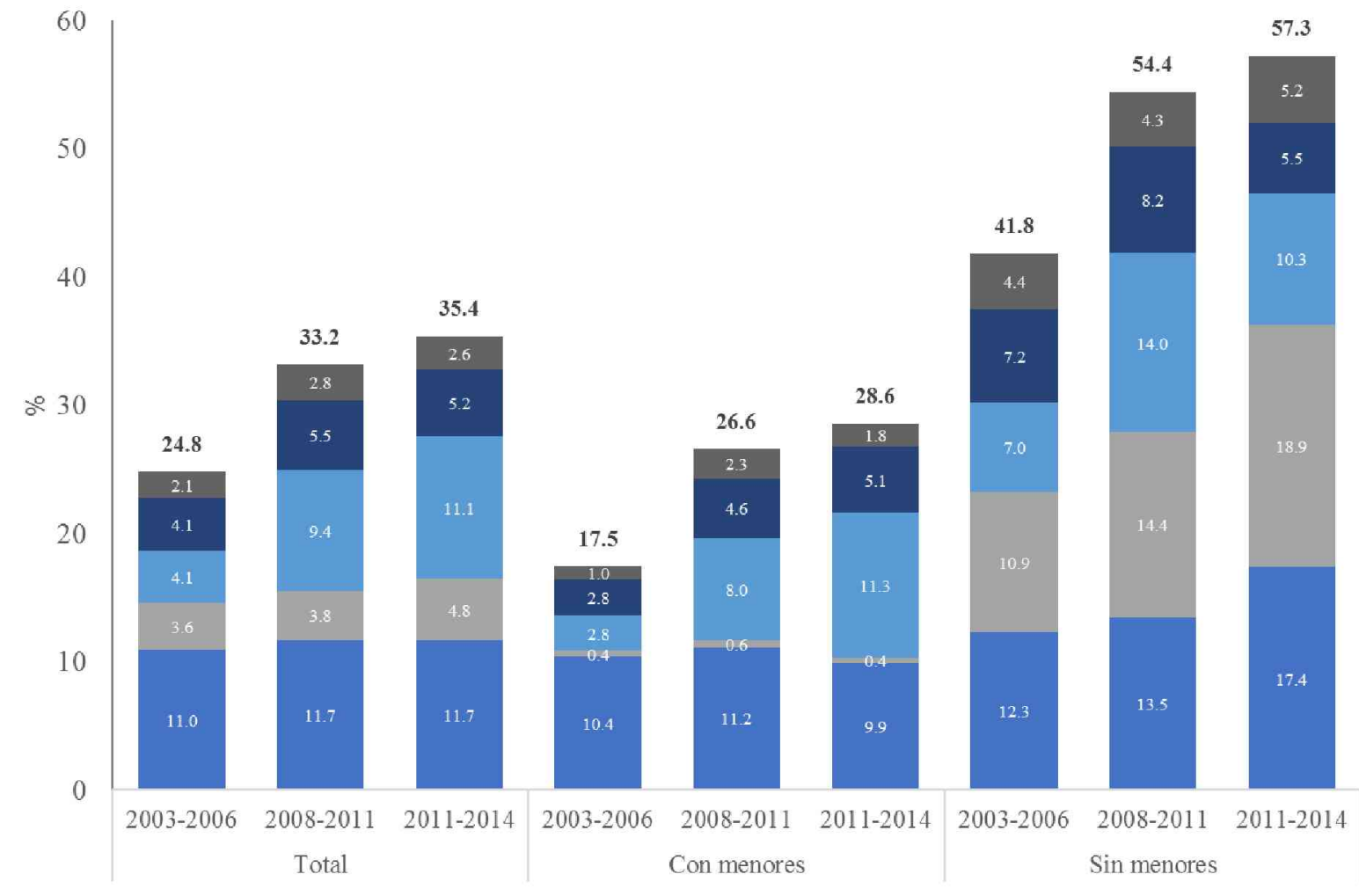

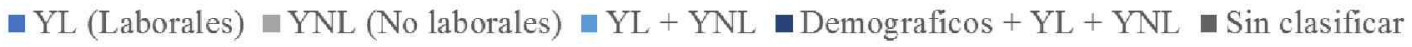

Fuente. Elaboración propia. Datos de la Encuesta Permanente de Hogares (EPH-INDEC).

Comparando según la composición del hogar, la diferencia en las tasas es relevante, pero cabe resaltar que la brecha entre hogares es mayor entre el primer período, que en los otros dos.

Para los hogares con presencia de menores del primer al segundo período se observa que la tasa aumenta en aproximadamente 9 p.p. debido tanto al aumento de ingresos laborales como por aumentos exclusivamente de ingresos no laborales y por la combinación de 
ambos aumentos. En el tercer período, baja la participación exclusivamente de los no laborales, pero aumenta en más de 3 p.p. la participación de la combinación de episodios.

En aquellos hogares sin menores es importante el ingreso no laboral en los últimos dos períodos, liderado por el aumento de las jubilaciones con un aumento acumulado del $37 \%$ entre los años 2009 y 2011.

En línea con el objetivo de esta investigación se despliega el análisis de las transferencias a través de los diferentes períodos. En esta década las transferencias por parte del Estado han ido aumentando, en especial aquellas destinadas a los hogares que tienen menores a cargo. En este sentido se pretende analizar el alcance de estas transferencias en las tasas de salida de la pobreza.

En el Figura 6.3 se observa que la probabilidad de salida asociada a este tipo de eventos ha aumentado a lo largo del tiempo para ambos tipos de hogares, pero en comparación, el primer resultado que se desprende es que la tendencia de aumento ha sido mayor para aquellos que tienen menores a cargo. En el último período, la probabilidad total asociada a transferencias es de 7,3\% para los hogares con menores, mientras que para los que no cuentan con presencia de menores fue en promedio alrededor del $2 \%$.

$\mathrm{Al}$ analizar la descomposición de la misma, se observan dos puntos relevantes:

- La probabilidad de ocurrencia de las transferencias aumenta entre períodos para los hogares con menores. En especial entre el primer y segundo período, esta probabilidad aumenta en tres veces. No ocurre lo mismo para los hogares sin menores la probabilidad de ocurrencia es baja, incluso aumenta en el segundo período, pero vuelve a disminuir en el tercero.

- Para los hogares con menores, la probabilidad condicional de salida dado la ocurrencia del evento, aumenta entre un período a otro, pero en la misma magnitud que la probabilidad de ocurrencia. En cambio, la probabilidad condicional para los hogares sin menores tiene un comportamiento diferente. En los primeros dos períodos se mantiene similar, pero aumenta a más del doble en el tercer período. 
Identificados estos dos puntos, es pertinente analizar el contexto de estos períodos. Respecto al primero, luego de la crisis macroeconómica a comienzo de la década del 2000, surge el paquete de transferencias que intenta apaciguar los daños que ha dejado a más de la mitad de la población por debajo de la línea de la pobreza.

Figura 6.1. Descomposición tasas de salida a la pobreza asociado a los eventos con transferencias, según composición del hogar. Sub períodos

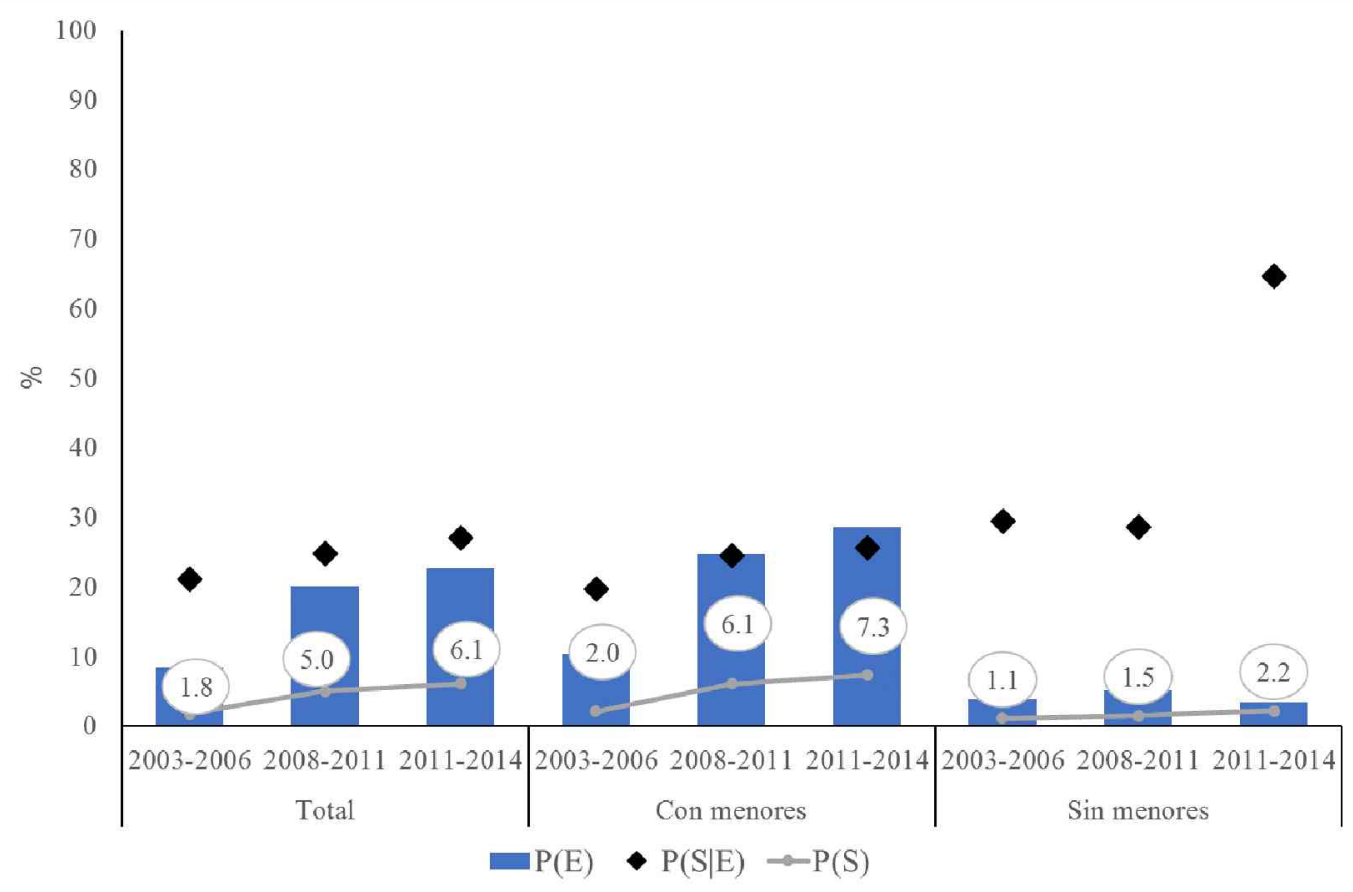

Fuente. Elaboración propia. Datos de la Encuesta Permanente de Hogares (EPH-INDEC).

El sistema de protección social se refuerza en el segundo período con la implementación de la Asignación Universal por Hijo (AUH) a fines del año 2009. Estas transferencias fueron una de las políticas más importantes del gobierno de turno, por su cobertura y por su magnitud (Gasparini y Cruces (2010)). Asimismo, sus primeros resultados han sido los esperados con una relación costo-efectividad muy razonable para reducir la pobreza extrema en los hogares con niños y adolescentes sin efectos estadísticamente significativos en el mercado laboral (Bertranou y Maurizzio (2012)). De esta manera se observa que el aumento de la probabilidad de ocurrencia en los hogares con menores se debe en parte al aumento de la AUH. Sin embargo, es importante notar que dentro de este conjunto también se incluyen otro tipo de transferencias, pero que no son tan relevantes como la AUH. Por 
este motivo se puede observar que en los hogares sin menores también hay aumento de transferencias, pero lejos de tener la misma intensidad.

Los resultados exponen que, a lo largo del período, el esfuerzo de las transferencias estuvo focalizado en los hogares con presencia de menores, sin embargo, se observa que la probabilidad condicional no es lo suficientemente alta, ya que, si bien ha aumentado a lo largo del tiempo, en especial entre el primer y segundo período, sólo un cuarto de los hogares que recibieron transferencias han logrado salir en el último período.

Por lo tanto, como resultado positivo para los hogares con menores, se observa una correcta focalización de las transferencias sobre estos hogares que ha ido aumentando a lo largo del tiempo, y una probabilidad condicional que también lo ha hecho, pero a menor ritmo. Sin embargo, el primer resultado que se obtiene es que, como es esperable, el paquete de transferencias por sí sólo no erradica la pobreza en los hogares, sino que debe ser un complemento a otras políticas que incentiven otros tipos de eventos, tales como los laborales. Además, se revela la magnitud de esta probabilidad condicional, que apenas llega a un tercio en el último año, agregando información complementaria a la evaluación el impacto de las transferencias.

\section{Indigencia}

\section{$\underline{\text { Período total }}$}

Respecto a la tasa de la indigencia los resultados son similares a los de la pobreza. El $82 \%$ de los hogares experimentó algún evento que potencialmente le permite salir del estado de indigencia y $54,6 \%$ ha logrado salir efectivamente de este estado. Al distinguir por tipo de hogar la brecha entre la tasa de salida es menor que en la pobreza. Este resultado es coherente, ya que las necesidades para salir de la indigencia son menores, y la vulnerabilidad de los hogares con menores es menor.

Al igual que en la pobreza, los eventos laborales son los que más se repiten en la descomposición de la tasa de salida. Sin embargo, ganan participación los eventos no laborales, que no sólo se observa en el conjunto de episodios de aumento exclusivo sino también en la combinación de ambos (Tabla 6.5). 


\section{Tabla 6.5 Descomposición de las tasas de salida de la indigencia según eventos. Período 2003-2014}

\begin{tabular}{|c|c|c|c|c|c|c|c|c|c|}
\hline \multirow[b]{2}{*}{ Eventos } & \multicolumn{3}{|c|}{ Total } & \multicolumn{3}{|c|}{ Con menores } & \multicolumn{3}{|c|}{ Sin menores } \\
\hline & $P(E)$ & $\mathrm{P}(\mathrm{S} / \mathrm{E})$ & $\mathrm{P}(\mathrm{S})$ & $\mathrm{P}(\mathrm{E})$ & $\mathrm{P}(\mathrm{S} / \mathrm{E})$ & $P(S)$ & $\mathrm{P}(\mathrm{E})$ & $\mathrm{P}(\mathrm{S} / \mathrm{E})$ & $P(S)$ \\
\hline 1.Eventos laborales & 29.1 & 68.6 & 20.0 & 29.6 & 64.6 & 19.1 & 27.5 & 83.6 & 23.0 \\
\hline 1.1.1 Aumento de cantidad de asalariados registrados sin entrada de un miembro ocupado al hogar & 1.5 & 98.1 & 1.5 & 1.8 & 97.8 & 1.7 & 0.7 & 100.0 & 0.7 \\
\hline 1.1.2 Aumento de cantidad de asalariados no registrados sin entrada de un miembro ocupado al hogar & 4.4 & 59.9 & 2.6 & 3.4 & 41.3 & 1.4 & 8.1 & 87.0 & 7.0 \\
\hline 1.1.3 Aumento de cantidad de no asalariados sin entrada de un miembro ocupado al hogar & 2.6 & 69.0 & 1.8 & 1.0 & 43.7 & 0.5 & 8.1 & 80.3 & 6.5 \\
\hline 1.2 Aumento en el ingreso horario de quienes están siempre ocupados & 6.4 & 60.4 & 3.8 & 7.0 & 56.3 & 4.0 & 4.0 & 85.5 & 3.4 \\
\hline 1.3 Aumento en la cantidad de horas trabajadas de los que están siempre ocupados & 1.9 & 31.3 & 0.6 & 2.3 & 32.5 & 0.7 & 0.5 & 13.7 & 0.1 \\
\hline 1.4 Aumento en el ingreso horario y en la cantidad de horas trabajadas (combinación 1.2 y 1.3 ) & 7.0 & 72.9 & 5.1 & 8.3 & 72.4 & 6.0 & 2.6 & 78.7 & 2.1 \\
\hline $\begin{array}{l}\text { 1.5 Aumento en la cantidad de ocupados y en el ingreso laboral de quienes están siempre ocupados (combinación } \\
\text { de todos los anteriores) }\end{array}$ & 5.3 & 84.9 & 4.5 & 5.8 & 83.5 & 4.9 & 3.4 & 92.8 & 3.2 \\
\hline 2. Eventos No Laborales & 10.9 & 49.1 & 5.4 & 7.6 & 26.6 & 2.0 & 22.6 & 75.6 & 17.1 \\
\hline 2.1 Aumento de ingresos por jubilación & 3.8 & 68.2 & 2.6 & 2.2 & 45.8 & 1.0 & 9.6 & 85.9 & 8.2 \\
\hline 2.2 Aumento de ingresos por politicas de transferencias & 3.7 & 11.7 & 0.4 & 4.2 & 12.2 & 0.5 & 2.0 & 7.3 & 0.1 \\
\hline 2.3 Aumento de otros ingresos no laborales (resto-jub-trans) & 3.4 & 68.1 & 2.3 & 1.3 & 41.2 & 0.5 & 11.0 & 78.9 & 8.7 \\
\hline 3. Aumento de ingreso laboral y aumento de trans fe rencias (ingreso no laboral) & 12.2 & 69.0 & 8.4 & 15.0 & 67.7 & 10.2 & 2.2 & 100.0 & 2.2 \\
\hline 4. Aumento de ingreso laboral y aumento de otros ingresos no laborales (no transferencias) & 4.4 & 81.4 & 3.6 & 4.1 & 82.3 & 3.4 & 5.5 & 79.2 & 4.3 \\
\hline 5. Combinación de aumento en ingresos laborales y aumentos de ambos anteriores & 1.3 & 89.8 & 1.2 & 1.6 & 89.1 & 1.4 & 0.4 & 100.0 & 0.4 \\
\hline 6. Demográfico puro (reducción en la cantidad de miembros sin aumento del ingreso total familiar) & 4.8 & 20.4 & 1.0 & 5.3 & 18.9 & 1.0 & 2.9 & 30.6 & 0.9 \\
\hline 7. Aumento en la cantidad ocupados & 1.8 & 74.2 & 1.4 & 1.7 & 68.6 & 1.1 & 2.5 & 87.1 & 2.2 \\
\hline 8. Reducción miembro + aumento en el ingreso total, incluido aumento de transferencias & 2.2 & 71.5 & 1.6 & 2.8 & 70.4 & 1.9 & 0.4 & 100.0 & 0.4 \\
\hline 9. Reducción miembro + aumento en el ingreso total, sin aumento de transferencias & 6.0 & 84.9 & 5.1 & 6.1 & 81.2 & 4.9 & 5.7 & 98.7 & 5.7 \\
\hline 10. Hogares sin clasificar & 9.6 & 73.1 & 7.0 & 9.7 & 67.0 & 6.5 & 9.2 & 95.1 & 8.8 \\
\hline Eventos con aume nto de trans fere ncias & 21.2 & 61.1 & 13.0 & 25.2 & 60.2 & 15.2 & 7.4 & 71.0 & 5.3 \\
\hline Hogares con Eventos (\%) & 82.4 & 66.2 & 54.6 & 83.5 & 61.9 & 51.7 & 78.8 & 82.2 & 64.8 \\
\hline Hogares sin eventos $(\%)$ & 17.6 & & & 16.5 & & & 21.2 & & \\
\hline Hogares totales $(\%)$ & 100.0 & & & 100.0 & & & 100.0 & & \\
\hline
\end{tabular}

Fuente. Elaboración propia. Datos de la Encuesta Permanente de Hogares (EPH-INDEC). 
En la indigencia entonces, los eventos no laborales por sí solos siguen siendo bajos, pero en comparación al análisis de la pobreza, toman mayor importancia. Particularmente se observa que la proporción de hogares que salen y que experimentaron sólo un aumento en las transferencias deja de ser nulo.

Sumando los eventos que involucran transferencias monetarias, se obtiene que para el período en general la probabilidad de salida asociada a algún aumento de transferencia monetaria por parte de Estado es del 13\% para el total de hogares, que corresponde a un $15,2 \%$ a los hogares con menores y un $5,3 \%$ al de los hogares sin menores.

La diferencia en la probabilidad total se debe a la mayor probabilidad de ocurrencia de estos eventos en los hogares con menores (en promedio es alrededor de $25 \%$ ) mientras que para los hogares sin menores es sólo del $7 \%$. Se advierte entonces la focalización de las transferencias en este tipo de hogares.

Sin embargo, la vulnerabilidad sigue siendo mayor en los primeros hogares que en los segundos, dado que la probabilidad condicional de salida es aún mayor para los hogares sin menores (aunque la diferencia de dichas probabilidades es menor que en la pobreza).

\section{$\underline{\text { Sub períodos }}$}

Realizando el mismo análisis anterior pero ahora para la indigencia, se observa que la probabilidad de ocurrencia de dichos eventos ha ido aumentando en los hogares con menores, y que dichas probabilidades son mayores que en la pobreza, es decir que las transferencias efectivamente llegan a los hogares de menores ingresos. Lo interesante a analizar aquí son de nuevo dos puntos:

- La probabilidad condicional en los hogares con menores es mayor en la indigencia que en la pobreza, específicamente es aproximadamente más del doble que en los períodos, pero sin embargo disminuye en el tercer período.

- Para los hogares sin menores, se observa la baja probabilidad de ocurrencia del evento, que se ha mantenido constante a lo largo del tiempo. Si bien su probabilidad condicional es alta, se deben a casos particulares de transferencias extraordinarias, no asociadas con las transferencias de programas sociales. 
En principio, que la probabilidad condicional sea mayor en la indigencia que en la pobreza es un resultado esperable, ya que la línea para salir de la indigencia es menor. Pero no es trivial la magnitud de estos resultados. En esta dirección, se observa que la condicionalidad en los primeros dos períodos es alta, tanto por los planes de emergencia post crisis, reforzado luego con la implementación de la AUH. En suma, el efecto fue casi tres veces más que en la pobreza. En el tercer período, esta probabilidad cae, producto del encarecimiento de los precios de los alimentos que impacta sobre la canasta básica alimentaria. Si bien las transferencias de la AUH se actualizan periódicamente con la inflación, se observa que el aumento del componente alimentos fue mayor al nivel general, generando que la actualización de las transferencias aumente menos que el valor de la CBA.

Figura 6.4. Descomposición tasas de salida a la indigencia asociado a los eventos con transferencias, según composición del hogar. Sub períodos

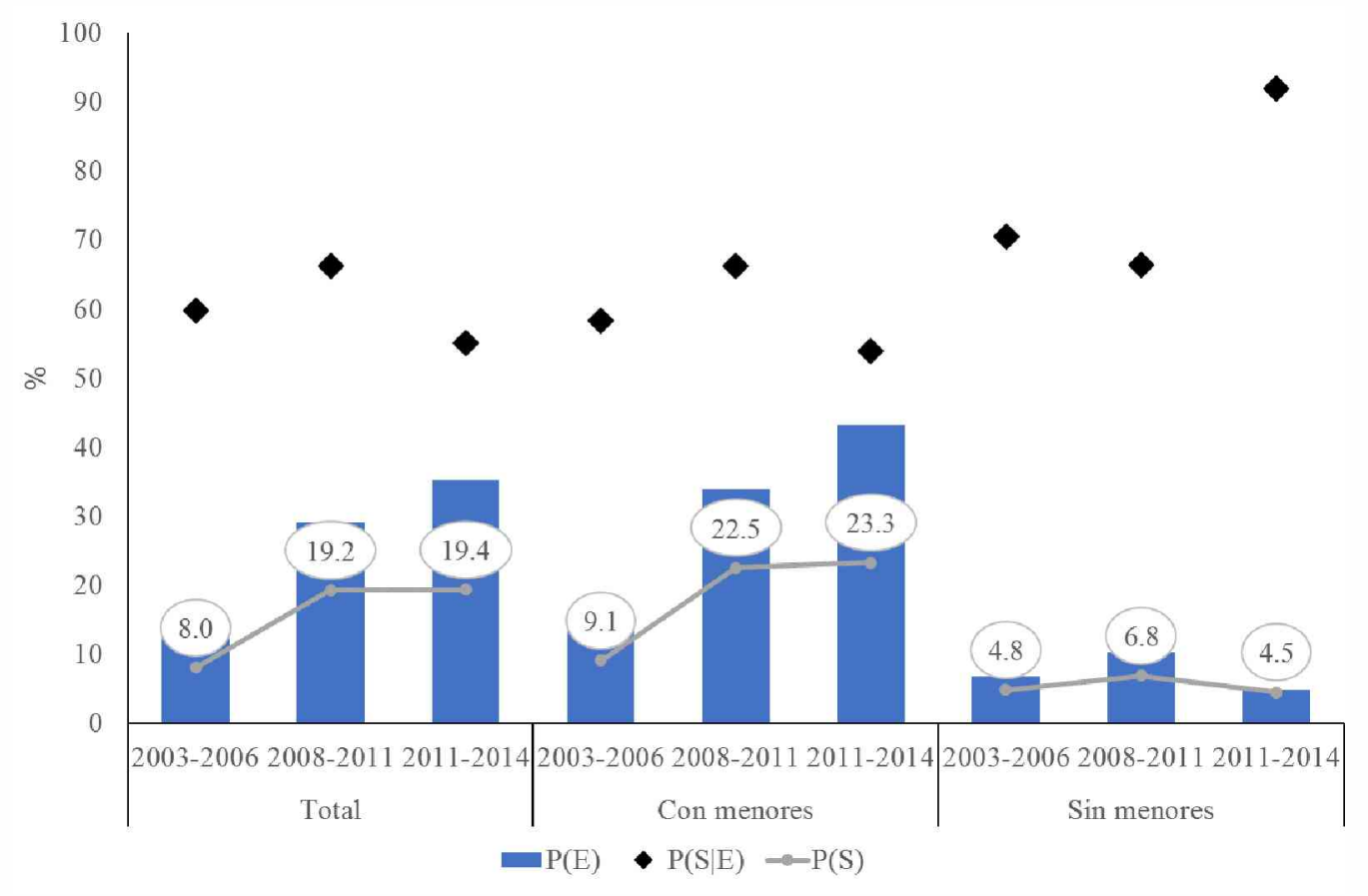

Fuente. Elaboración propia. Datos de la Encuesta Permanente de Hogares (EPH-INDEC). 


\subsection{Análisis de sensibilidad}

En esta sección se realiza un análisis de sensibilidad ante cambios en la línea de pobreza, a partir de considerar dos líneas alternativas, la primera un $10 \%$ superior y la segunda $10 \%$. El objetivo es ver si los resultados se mantienen cuando no se consideran a los hogares que entran y salen de la pobreza por estar demasiado cerca de la línea.

Con una línea más exigente, es decir de un $10 \%$ superior las tasas de salida deberían ser menores. Los resultados se exponen en la tabla 6.6. Efectivamente, disminuyen en aproximadamente 2 p.p. para el total de hogares durante todo el período. Distinguiendo por composición del hogar, la caída en las transiciones de salida de la pobreza es mayor para los hogares sin menores, indicando que hay más cantidad de este tipo de hogares que está por sobre la línea, pero lo suficientemente cerca para caer.

Teniendo en cuenta una línea de pobreza un $10 \%$ inferior, las tasas de salida son 2 p.p. mayores para el total de los hogares (tabla 6.7). Respecto a la distribución de las tasas según los episodios, se observa que los cambios son similares para los dos tipos de hogar, lo que indica que las conclusiones anteriores son robustas al cambio de la línea de la pobreza. 
Tabla 6.6 Análisis de Sensibilidad Línea 10\% superior. Descomposición de las tasas de salida de la pobreza según eventos.

\begin{tabular}{|c|c|c|c|c|c|c|c|c|c|}
\hline \multirow[b]{2}{*}{ Eventos } & \multicolumn{3}{|c|}{ Total } & \multicolumn{3}{|c|}{ Con menores } & \multicolumn{3}{|c|}{ Sin menores } \\
\hline & $\mathrm{P}(\mathrm{E})$ & $\mathrm{P}(\mathrm{S} / \mathrm{E})$ & $\mathrm{P}(\mathrm{S})$ & $\mathrm{P}(\mathrm{E})$ & $\mathrm{P}(\mathrm{S} / \mathrm{E})$ & $\mathrm{P}(\mathrm{S})$ & $\mathrm{P}(\mathrm{E})$ & $\mathrm{P}(\mathrm{S} / \mathrm{E})$ & $\mathrm{P}(\mathrm{S})$ \\
\hline 1.Eventos laborales & 32.3 & 33.3 & 10.8 & 36.3 & 28.5 & 10.4 & 22.0 & 53.7 & 11.8 \\
\hline 1.1.1 Aumento de cantidad de asalariados registrados sin entrada de un miembro ocupado al hogar & 0.9 & 40.2 & 0.4 & 1.1 & 30.3 & 0.3 & 0.5 & 97.4 & 0.5 \\
\hline 1.1.2 Aumento de cantidad de asalariados no registrados sin entrada de un miembro ocupado al hogar & 2.2 & 22.0 & 0.5 & 2.0 & 8.1 & 0.2 & 2.5 & 50.8 & 1.3 \\
\hline 1.1.3 Aumento de cantidad de no asalariados sin entrada de un miembro ocupado al hogar & 1.1 & 25.3 & 0.3 & 0.6 & 14.9 & 0.1 & 2.4 & 31.9 & 0.8 \\
\hline 1.2 Aumento en el ingreso horario de quienes están siempre ocupados & 12.3 & 32.3 & 4.0 & 14.4 & 28.4 & 4.1 & 7.0 & 52.7 & 3.7 \\
\hline 1.3 Aumento en la cantidad de horas trabajadas de los que están siempre ocupados & 3.2 & 13.5 & 0.4 & 3.7 & 8.4 & 0.3 & 1.9 & 39.2 & 0.7 \\
\hline 1.4 Aumento en el ingreso horario y en la cantidad de horas trabajadas (combinación 1.2 y 1.3 ) & 7.7 & 37.9 & 2.9 & 8.9 & 33.5 & 3.0 & 4.6 & 59.9 & 2.7 \\
\hline $\begin{array}{l}\text { 1.5 Aumento en la cantidad de ocupados y en el ingreso laboral de quienes están siempre ocupados (combinación } \\
\text { de todos los anteriores) }\end{array}$ & 5.0 & 46.9 & 2.3 & 5.7 & 42.4 & 2.4 & 3.2 & 67.3 & 2.1 \\
\hline 2. Eventos No Laborales & 11.8 & 34.5 & 4.1 & 5.6 & 8.1 & 0.5 & 27.4 & 48.2 & 13.2 \\
\hline 2.1 Aumento de ingresos por jubilación & 7.3 & 43.9 & 3.2 & 2.0 & 13.9 & 0.3 & 20.8 & 51.1 & 10.6 \\
\hline 2.2 Aumento de ingresos por políticas de transferencias & 2.1 & 2.0 & 0.0 & 2.7 & 0.6 & 0.0 & 0.8 & 14.2 & 0.1 \\
\hline 2.3 Aumento de otros ingresos no laborales (resto-jub-trans) & 2.4 & 34.8 & 0.8 & 1.0 & 16.5 & 0.2 & 5.8 & 42.8 & 2.5 \\
\hline 3. Aumento de ingreso laboral y aumento de trans ferencias (ingreso no laboral) & 8.3 & 22.8 & 1.9 & 10.9 & 22.5 & 2.5 & 1.5 & 28.1 & 0.4 \\
\hline 4. Aumento de ingreso laboral y aumento de otros ingresos no laborales (no transferencias) & 7.3 & 58.4 & 4.2 & 6.0 & 43.5 & 2.6 & 10.4 & 80.3 & 8.4 \\
\hline 5. Combinación de aumento en ingresos laborales y aumentos de ambos anteriores & 1.3 & 26.0 & 0.3 & 1.7 & 24.0 & 0.4 & 0.3 & 62.4 & 0.2 \\
\hline 6. Demográfico puro (reducción en la cantidad de miembros sin aumento del ingreso total familiar) & 5.2 & 16.3 & 0.9 & 5.5 & 14.9 & 0.8 & 4.7 & 20.4 & 1.0 \\
\hline 7. Aumento en la cantidad ocupados & 0.9 & 19.3 & 0.2 & 0.9 & 12.1 & 0.1 & 0.9 & 36.6 & 0.3 \\
\hline 8. Reducción miembro + aumento en el ingreso total, incluido aumento de transferencias & 1.0 & 35.8 & 0.4 & 1.3 & 33.6 & 0.4 & 0.2 & 65.3 & 0.2 \\
\hline 9. Reducción miembro + aumento en el ingreso total, sin aumento de transferencias & 5.0 & 57.6 & 2.9 & 4.7 & 45.1 & 2.1 & 5.9 & 82.7 & 4.9 \\
\hline 10. Hogares sin clasificar & 6.9 & 33.1 & 2.3 & 6.5 & 21.5 & 1.4 & 7.9 & 57.5 & 4.5 \\
\hline Eventos con aume nto de trans fe re ncias & 13.6 & 20.6 & 2.8 & 17.5 & 19.6 & 3.4 & 3.7 & 32.0 & 1.2 \\
\hline Hogares con Eventos (\%) & 80.0 & 34.8 & 27.9 & 79.4 & 26.6 & 21.2 & 81.3 & 55.2 & 44.9 \\
\hline Hogares sin eventos $(\%)$ & 20.0 & & & 20.6 & & & 18.7 & & \\
\hline Hogares totales (\%) & 100.0 & & & 100.0 & & & 100.0 & & \\
\hline
\end{tabular}

Fuente. Elaboración propia. Datos de la Encuesta Permanente de Hogares (EPH-INDEC) 
Tabla 6.7 Análisis de Sensibilidad. Línea 10\% inferior. Descomposición de las tasas de salida de la pobreza según eventos.

\begin{tabular}{|c|c|c|c|c|c|c|c|c|c|}
\hline \multirow[b]{2}{*}{ Eventos } & \multicolumn{3}{|c|}{ Total } & \multicolumn{3}{|c|}{ Con menores } & \multicolumn{3}{|c|}{ Sin menores } \\
\hline & $\mathrm{P}(\mathrm{E})$ & $\mathrm{P}(\mathrm{S} / \mathrm{E})$ & $\mathrm{P}(\mathrm{S})$ & $\mathrm{P}(\mathrm{E})$ & $\mathrm{P}(\mathrm{S} / \mathrm{E})$ & $\mathrm{P}(\mathrm{S})$ & $\mathrm{P}(\mathrm{E})$ & $\mathrm{P}(\mathrm{S} / \mathrm{E})$ & $\mathrm{P}(\mathrm{S})$ \\
\hline 1.Eventos laborales & 31.9 & 37.1 & 11.8 & 35.0 & 32.2 & 11.2 & 23.1 & 58.4 & 13.5 \\
\hline 1.1.1 Aumento de cantidad de asalariados registrados sin entrada de un miembro ocupado al hogar & 1.1 & 52.6 & 0.6 & 1.2 & 44.4 & 0.5 & 0.6 & 100.0 & 0.6 \\
\hline 1.1.2 Aumento de cantidad de asalariados no registrados sin entrada de un miembro ocupado al hogar & 2.6 & 27.8 & 0.7 & 2.4 & 14.6 & 0.3 & 3.1 & 57.0 & 1.8 \\
\hline 1.1.3 Aumento de cantidad de no asalariados sin entrada de un miembro ocupado al hogar & 1.2 & 29.2 & 0.3 & 0.5 & 11.0 & 0.1 & 3.2 & 36.9 & 1.2 \\
\hline 1.2 Aumento en el ingreso horario de quienes están siempre ocupados & 10.9 & 35.3 & 3.9 & 12.5 & 30.7 & 3.8 & 6.4 & 60.7 & 3.9 \\
\hline 1.3 Aumento en la cantidad de horas trabajadas de los que están siempre ocupados & 3.2 & 14.2 & 0.5 & 3.7 & 10.0 & 0.4 & 2.0 & 36.2 & 0.7 \\
\hline 1.4 Aumento en el ingreso horario y en la cantidad de horas trabajadas (combinación 1.2 y 1.3 ) & 7.8 & 41.8 & 3.3 & 9.0 & 38.0 & 3.4 & 4.6 & 63.1 & 2.9 \\
\hline $\begin{array}{l}\text { 1.5 Aumento en la cantidad de ocupados y en el ingreso laboral de quienes están siempre ocupados (combinación } \\
\text { de todos los anteriores) }\end{array}$ & 5.1 & 51.6 & 2.6 & 5.7 & 46.9 & 2.7 & 3.2 & 75.8 & 2.5 \\
\hline 2. Eventos No Laborales & 11.1 & 35.3 & 3.9 & 5.9 & 7.5 & 0.4 & 25.9 & 53.4 & 13.9 \\
\hline 2.1 Aumento de ingresos por jubilación & 6.2 & 45.0 & 2.8 & 2.0 & 10.8 & 0.2 & 18.1 & 55.8 & 10.1 \\
\hline 2.2 Aumento de ingresos por políticas de transferencias & 2.4 & 1.5 & 0.0 & 2.8 & 0.5 & 0.0 & 1.1 & 8.8 & 0.1 \\
\hline 2.3 Aumento de otros ingresos no laborales (resto-jub-trans) & 2.5 & 43.1 & 1.1 & 1.1 & 19.3 & 0.2 & 6.7 & 54.1 & 3.6 \\
\hline 3. Aumento de ingreso laboral y aumento de trans fe re ncias (ingreso no laboral) & 9.3 & 31.6 & 2.9 & 11.9 & 30.6 & 3.6 & 1.9 & 49.6 & 1.0 \\
\hline 4. Aumento de ingreso laboral y aumento de otros ingresos no laborales (no transferencias) & 6.5 & 59.9 & 3.9 & 5.4 & 46.3 & 2.5 & 9.9 & 81.2 & 8.1 \\
\hline 5. Combinación de aumento en ingresos laborales y aumentos de ambos anteriores & 1.5 & 43.5 & 0.7 & 1.9 & 42.1 & 0.8 & 0.3 & 71.0 & 0.2 \\
\hline 6. Demográfico puro (reducción en la cantidad de miembros sin aumento del ingreso total familiar) & 5.5 & 15.1 & 0.8 & 5.7 & 12.9 & 0.7 & 4.9 & 22.5 & 1.1 \\
\hline 7. Aumento en la cantidad ocupados & 1.0 & 21.8 & 0.2 & 1.0 & 16.3 & 0.2 & 1.2 & 35.3 & 0.4 \\
\hline 8. Reducción miembro + aumento en el ingreso total, incluido aumento de transferencias & 1.2 & 49.1 & 0.6 & 1.5 & 46.6 & 0.7 & 0.3 & 86.6 & 0.3 \\
\hline 9. Reducción miembro + aumento en el ingreso total, sin aumento de transferencias & 5.5 & 64.0 & 3.5 & 5.1 & 52.2 & 2.7 & 6.6 & 90.7 & 6.0 \\
\hline 10. Hogares sin clasificar & 7.1 & 36.4 & 2.6 & 7.0 & 27.7 & 1.9 & 7.6 & 59.6 & 4.5 \\
\hline Eventos con aumento de trans fe re ncias & 15.4 & 28.9 & 4.5 & 19.2 & 27.9 & 5.3 & 4.7 & 40.5 & 1.9 \\
\hline Hogares con Eventos (\%) & 80.7 & 38.4 & 31.0 & 80.4 & 30.9 & 24.8 & 81.7 & 59.8 & 48.8 \\
\hline Hogares sin eventos $(\%)$ & 19.3 & & & 19.6 & & & 18.3 & & \\
\hline Hogares totales (\%) & 100.0 & & & 100.0 & & & 100.0 & & \\
\hline
\end{tabular}

Fuente. Elaboración propia. Datos de la Encuesta Permanente de Hogares (EPH-INDEC). 


\section{Conclusiones}

Este trabajo estudia las transiciones de la pobreza, analizando especialmente las tasas de salida de la pobreza y de la indigencia para hogares con y sin presencia de menores, en Argentina durante el período 2003-2014.

En primer lugar, si bien, el período se caracteriza por tener una tendencia decreciente en los niveles de pobreza, siempre existe un porcentaje no despreciable de hogares que entra en ella.

Del análisis se revela que las tasas de salida se asocian en mayor proporción a los eventos laborales. Entre ellos, los más concurrentes son los que involucran aumentos en los ingresos laborales de los hogares, ya sea por aumentos de ingreso horario, de horas trabajadas o por una combinación de ambos. Además, en relación a los episodios que captan la inserción laboral, se observa que es mayor la probabilidad de inserción en el mercado informal, sin embargo, la probabilidad condicional de salida es mayor para los hogares con presencia de menores que logran insertar a sus miembros en el sector formal. De aquí la importancia, de alentar el desarrollo del mercado laboral formal como motor para aumentar las tasas de salida y por tanto la reducción de la pobreza.

Al distinguir por la composición del hogar, se observa una amplia brecha entre los hogares con y sin presencia de menores. Específicamente, las tasas de salida del segundo grupo son más del doble que la del primero, dando cuenta de que la estructura del hogar es un factor relevante en las transiciones de la pobreza.

En este sentido, el gobierno de Argentina ha dedicado esfuerzos para atender a este grupo de la población a través de transferencias monetarias que ayuden a compensar los gastos extras que tienen las familias con menores a cargo. A lo largo del período, se observa que aumenta la probabilidad de los hogares con presencia de menores de recibir transferencias gubernamentales. Sin embargo, también se revela un resultado esperable, sólo el hecho de recibir una transferencia monetaria no resulta suficiente para salir de la pobreza.

Los resultados son más interesantes para la indigencia. La probabilidad de salir de la indigencia dado que el hogar recibió una transferencia es más del doble que la probabilidad 
de salir de la pobreza. Esto sugiere que el efecto esperado de las transferencias monetarias, sí cumplen un papel relevante en las tasas de salida de la pobreza extrema.

Por lo tanto, del estudio se desprende que, si bien existe una focalización de las transferencias a los hogares más vulnerables en este período, gran parte de los hogares no logra salir de la pobreza moderada. No obstante, estas transferencias se asocian en mayor proporción en las tasas de salida en la indigencia, donde más de la mitad de los hogares con menores logra salir dado que recibió una transferencia gubernamental.

Resulta importante ser consciente del alcance e impacto de las políticas públicas sobre el bienestar social, teniendo presente que toda política de protección social requiere una continua evaluación que permita reflexionar sobre su ajuste, para que se convierta parte de una política pública integral. En este caso si bien las transferencias desde el Estado son un buen complemento para aliviar a los hogares, deben encuadrarse dentro de una política social que promuevan el desarrollo del hogar, tales como programas orientados a la inserción laboral en el sector formal. 


\section{Bibliografía}

Bane, M. y D, Ellwood. (1986). "Slipping into and out of poverty: the dynamics of spells". Journal of Human Resources, 21 (1).

Beccaria, L. (2017). "La pobreza por ingresos y su comparación en el tiempo" Alquimias económicas. Disponible en https://alquimiaseconomicas.com/2017/07/19/la-pobreza-por-ingresosv-su-comparacion-en-el-tiempo/

Beccaria, L. y Maurizio. R. (2006). "Factors associated to poverty mobility in Greater Buenos Aires". Universidad Nacional de General Sarmiento, Argentina.

Beccaria, L., Maurizio, R., Vázquez, G., y Espro, M. (2015). "Factors Associated with Poverty and Indigence Mobility in Five Latin American Countries. Measurement of Poverty, Deprivation, and Economic Mobility" Research on Economic Inequality, Volume 23, Emerald Group Publishing Limited, 23, 71-107.

Beccaria, L., Maurizio, R., Fernández, A. L., Monsalvo, P., y Álvarez, M. (2013). "Urban poverty and labor market dynamics in five Latin American countries: 2003-2008". The Journal of Economic Inequality, 11(4), 555-580.

Bertranou, F. y Maurizio, R. (2012). "Transferencias Monetarias para Niños y Adolescentes en Argentina: Características y Cobertura de un Sistema con Tres Componentes" (No. 30). International Policy Centre for Inclusive Growth.

Beccaria, L., Maurizio, R., Fernandez, A. L., Monsalvo, A. P., \& Alvarez, M. (2011). "Dynamics of poverty, labor market and public policies in Latin America". PEP PMMA, 5.

Beccaria, L., Esquivel, V., \& Maurizio, R. (2005). Empleo, salarios y equidad durante la recuperación reciente en la Argentina. Desarrollo Económico, 235-262.

Cantó, O., C. del Río y C. Gradín. (2006). "What helps households with children in leaving poverty? Evidence from Spain in contrast with other EU countries, Working Paper 2006-24, Departamento de Economía Aplicada, University of Vigo, España.

Cappellari, L. y S. Jenkins. (2002). "Modelling low income transitions". Papers 504, Institute for the Study of Labor (IZA).

Cruces, G. y Q. Wodon. (2003). "Transient and chronic poverty in turbulent times: Argentina 19952002" Economic Bulletin, Vol. 9, No 3

Devicienti, F. (2001). "Poverty persistence in Britain: a multivariate analysis using the BHPS, 19911997”. ISER Working paper 2001-02, University of Essex, Colchester.

Gasparini, L., \& Cruces, G. (2010). "Las Asignaciones Universales por Hijo: impacto, discusión y alternativas". Documentos de Trabajo del CEDLAS.

Garganta, S., \& Gasparini, L. (2012). El impacto de un programa social sobre la informalidad laboral: el caso de la AUH en Argentina. Documento de trabajo, (133). 
Gasparini, L., Cicowiez, M., \& Escudero, W. S. (2013). Pobreza y desigualdad en América Latina. Conceptos, herramientas y aplicaciones. Capítulo 4. Buenos Aires.

Jenkins, S. P., \& Schluter, C. (2003). Why are child poverty rates higher in Britain than in Germany? A longitudinal perspective. Journal of Human Resources, 38(2), 441-465.

Jenkins, S. P., Rigg, J. A., \& Devicienti, F. (2001). The dynamics of poverty in Britain. Corporate Document Services for the Department for Work and Pensions.

Lillard, L. y R. Willis (1978). "Dynamic aspects of earnings mobility”, Econometrica 46: 985-1012.

Maurizio, R., Perrot, B. y Villafañe, S. (2008). "Dinámica de la pobreza y mercado de trabajo en Argentina post-convertibilidad" Proyecto PNUD ARG/04/034, Argentina.

Paz, J. (2005) "Pobres, pobres, cada vez más pobres. Una visión global de la pobreza", en Mercado de trabajo y Equidad en Argentina, Beccaria, L. y R. Maurizio (eds). Bs. As., Prometeo. 


\section{Anexo}

\subsection{Características de los hogares pool paneles}

\begin{tabular}{|c|c|c|c|c|c|c|c|c|}
\hline & \multicolumn{4}{|c|}{ Corte Transversal } & \multicolumn{4}{|c|}{ Paneles } \\
\hline & $\begin{array}{c}\text { Comp. } \\
\text { total }\end{array}$ & $\begin{array}{c}\text { No } \\
\text { pobres }\end{array}$ & Pobres & Total & $\begin{array}{c}\text { Comp. } \\
\text { total }\end{array}$ & $\begin{array}{c}\text { No } \\
\text { pobres }\end{array}$ & Pobres & Total \\
\hline \multicolumn{9}{|l|}{ Niveles educativos } \\
\hline Hasta primaria completa & $55 \%$ & $60 \%$ & $40 \%$ & $100 \%$ & $56 \%$ & $60 \%$ & $40 \%$ & $100 \%$ \\
\hline Secundaria Incompleta y completa & $31 \%$ & $80 \%$ & $20 \%$ & $100 \%$ & $30 \%$ & $81 \%$ & $19 \%$ & $100 \%$ \\
\hline Universitaria Incompleta y completa & $15 \%$ & $94 \%$ & $6 \%$ & $100 \%$ & $13 \%$ & $93 \%$ & $7 \%$ & $100 \%$ \\
\hline Total & $100 \%$ & $71 \%$ & $29 \%$ & $100 \%$ & $100 \%$ & $71 \%$ & $29 \%$ & $100 \%$ \\
\hline \multicolumn{9}{|l|}{ Edad } \\
\hline Edad $<25$ & $4 \%$ & $64 \%$ & $36 \%$ & $100 \%$ & $4 \%$ & $61 \%$ & $39 \%$ & $100 \%$ \\
\hline $25<=\operatorname{Edad}<=40$ & $31 \%$ & $65 \%$ & $35 \%$ & $100 \%$ & $30 \%$ & $64 \%$ & $36 \%$ & $100 \%$ \\
\hline $41<=$ Edad $<=65$ & $43 \%$ & $68 \%$ & $32 \%$ & $100 \%$ & $42 \%$ & $68 \%$ & $32 \%$ & $100 \%$ \\
\hline Edad $>65$ & $22 \%$ & $85 \%$ & $15 \%$ & $100 \%$ & $24 \%$ & $86 \%$ & $14 \%$ & $100 \%$ \\
\hline Total & $100 \%$ & $71 \%$ & $29 \%$ & $100 \%$ & $100 \%$ & $71 \%$ & $29 \%$ & $100 \%$ \\
\hline \multicolumn{9}{|l|}{ Sexo } \\
\hline Mujer & $35 \%$ & $73 \%$ & $27 \%$ & $100 \%$ & $34 \%$ & $74 \%$ & $26 \%$ & $100 \%$ \\
\hline Hombre & $65 \%$ & $70 \%$ & $30 \%$ & $100 \%$ & $66 \%$ & $70 \%$ & $30 \%$ & $100 \%$ \\
\hline Total & $100 \%$ & $71 \%$ & $29 \%$ & $100 \%$ & $100 \%$ & $71 \%$ & $29 \%$ & $100 \%$ \\
\hline \multicolumn{9}{|l|}{ Categoría ocupacional } \\
\hline Asalariados registrados & $33 \%$ & $84 \%$ & $16 \%$ & $100 \%$ & $33 \%$ & $83 \%$ & $17 \%$ & $100 \%$ \\
\hline Asalariados no registrados & $16 \%$ & $51 \%$ & $49 \%$ & $100 \%$ & $15 \%$ & $51 \%$ & $49 \%$ & $100 \%$ \\
\hline Cuentapropistas & $14 \%$ & $60 \%$ & $40 \%$ & $100 \%$ & $13 \%$ & $58 \%$ & $42 \%$ & $100 \%$ \\
\hline Patrones & $3 \%$ & $86 \%$ & $14 \%$ & $100 \%$ & $3 \%$ & $84 \%$ & $16 \%$ & $100 \%$ \\
\hline Desocupados & $4 \%$ & $32 \%$ & $68 \%$ & $100 \%$ & $4 \%$ & $32 \%$ & $68 \%$ & $100 \%$ \\
\hline Inactivos & $30 \%$ & $75 \%$ & $25 \%$ & $100 \%$ & $32 \%$ & $77 \%$ & $23 \%$ & $100 \%$ \\
\hline Total & $100 \%$ & $71 \%$ & $29 \%$ & $100 \%$ & $100 \%$ & $71 \%$ & $29 \%$ & $100 \%$ \\
\hline \multicolumn{9}{|l|}{ Composición del Hogar } \\
\hline Hogares unipersonales $=>65$ & $9 \%$ & $94 \%$ & $6 \%$ & $100 \%$ & $10 \%$ & $95 \%$ & $5 \%$ & $100 \%$ \\
\hline Hogares unipersonales $=<65$ & $12 \%$ & $88 \%$ & $12 \%$ & $100 \%$ & $12 \%$ & $87 \%$ & $13 \%$ & $100 \%$ \\
\hline Hogares compuestos jefe $=>65$ sin menores & $11 \%$ & $85 \%$ & $15 \%$ & $100 \%$ & $12 \%$ & $84 \%$ & $16 \%$ & $100 \%$ \\
\hline Hogares compuestos jefe $=<65$ sin menores & $21 \%$ & $81 \%$ & $19 \%$ & $100 \%$ & $20 \%$ & $80 \%$ & $20 \%$ & $100 \%$ \\
\hline Hogares compuestos con menores & $48 \%$ & $55 \%$ & $45 \%$ & $100 \%$ & $46 \%$ & $54 \%$ & $46 \%$ & $100 \%$ \\
\hline Total & $100 \%$ & $71 \%$ & $29 \%$ & $100 \%$ & $100 \%$ & $71 \%$ & $29 \%$ & $100 \%$ \\
\hline \% hogares con menores & & $37 \%$ & $74 \%$ & & & $35 \%$ & $73 \%$ & \\
\hline
\end{tabular}




\subsection{IPC empalme 8 provincias}

\begin{tabular}{|c|c|c|c|c|c|c|c|c|}
\cline { 2 - 8 } \multicolumn{1}{c|}{} & Jujuy & Salta & Córdoba & San Luis & Santa Fe & $\begin{array}{c}\text { Tierra del } \\
\text { Fuego }\end{array}$ & Neuquén & CABA \\
\hline 2006 & Octubre & Octubre & & Octubre & & & Octubre & \\
\hline 2007 & & & & & & & & \\
\hline 2008 & & & & & & & & \\
\hline 2009 & & & & & & & & \\
\hline 2010 & & & & & & & & \\
\hline 2011 & & & & & & & & \\
\hline 2012 & & & & & Julio & & & \\
\hline 2013 & & & Agosto & & & Junio & & \\
\hline 2014 & & & & & & & & \\
\hline 2015 & & & & & & Noviembre & & \\
\hline
\end{tabular}

A partir de las tasas de variación mensual, se obtiene una variación para cada categoría $\dot{t}$ como el promedio simple de las tasas de variación por provincia $j$ :

$\Delta I P C_{t}^{i}=\frac{1}{J} \sum_{j} \Delta I P C_{t}^{i j}$

Utilizando los ponderadores $\alpha_{i}$ por categoría del IPC-GBA INDEC base 2004/05 se reconstruye la tasa de variación mensual del índice:

$\triangle I P C_{t}=\alpha_{i} \triangle I P C_{t}^{i}$ 